\title{
ON OPTIMAL SHAFE DESIGN OF SYSTEMS EOVERNED BY MIXED DIRICHLET-SIGNORINI BOUNDARY VALUE PROBLEMS
}

J. Haslinger and ?. Neittaanmäki

Preprint 22

June 1983

University of Jyväskylä, Jyväskylä 2017

978-951-39-7030-7 PDF

ISSN 0780-8569 
ON OPTIMAL SHAPE DESIGN OF SYSTEMS GOVERNED BY MIXED DIRICHLET-SIGNORINI BOUNDARY VALUE PROBLEMS

]. Haslinger and

Faculty of Mathematics and Physics

Charles University KAM MFF UK, Malostranské 2/25 11800 Prague Czechoslovakia
P. Neittaanmäki

Department of Mathematics University of Jyväskylä

Seminarinkatu 15 SF-40100 Jyväskylä 10

Finland

\section{Abstract}

A problem for finding optimal shape for systems governed by the mixed unilateral boundary value problem of DirichletSignorini-type is considered. Conditions for the solvability of the problem are stated, when variational inequality formulation and penalty method is used for solving the state problem in question. The asymptotic relation of design problems based on these two formulations is presented. The optimal shape design problem is discretized by means of finite element method. The convergence results for the approximation are proved. The discretized versions are then formulated as a nonlinear programming problem. Results of practical computations of the problem in question are reported. 
1. INTROOUCTION

During the past 15 years, optimization theory has been developed for the optimal design of many structural and mechanical systems. Generally speaking, an optimal shape design problem is an optimization problem that involves a function satisfying certain relations cordinary or partial differential equations, e.g.) and optimization variables with geometrical structure of the problem in question. Optimal shape design problems, where the state problem is modelled by a partial differential equation, have been discussed widely in engineering literature [13, 14, 21, 22, 23], see especially the article of Haug [13], where a representative survey of appeared contributions can be found. Mathematical theory of such kind of problems, including the theory of their approximation by finite differences or finite elements, has been developed during the last ten years mainiy by the French school of applied mathematics $[3,4,5,6,17,25,29]$. See also [1] and [28].

In practice, we often meet problems the behaviour af which is descrited by variational inequalities (see [7]). A natural question arises: what happens if the state problem is now given by these inequalities? Mathematical theory of problems with controlled right hand side has been given e.g. in [24], [17]. In the meantime, shape sensitivity analysis of design problems (control of coefficients) has been given in [26, 27]. A relatively small number of papers is devoted to optimal shape design problems with state inequalities. We mention here papers [15, 16] where the existence of a solution as well as the approximation are studied. It is well known that the relation between admissible designs and the corresponding solution of state inequality is not differentiable everywhere. From this point of view, some specific optimization approach has to be used for numerical realization. To overcome this 
difficulty in [10, 11] an alternative way has been proposed: instead of variational inequality (associated to a unilateral boundary value problem) a family of penalized variational equations is assumed. It can be shown (see Theorem 2.3, in [10]) that the corresponding optimal designs (associated with penalized problems) are close (in an appropriate sense) to an optimal design associated with the variational inequality formulation.

The aim of the present paper is to study the finite element approximation of our penalized design problems. The paper is organized as follows:

1. Introduction

2. Statement of the optimal shape design problem

3. Approximation of the penalized problem

4. Numerical realization of optimal shape design problem

4.1 Construction of moving triangular grid

4.2 Algebraic formulation of discretized shape design problem

4.3 Computation of the gradient for the cost functional 4.4 Algorithms for solving Problem ( $P)_{h}$

5. Remarks on alternative methods

5.1 Dual formulation of the state problem

5.2 Adjoint state for the penalized problem

6. Numerical examples

References

The problem in question can be regarded as a model example of the contact problem between an elastic body and a rigid foundation, which will be studied in a forthcoming paper [12]. 
2. STATEMENT OF THE OPTIMAL SHAPE, DESIGN PROBLEM

Let us consider domains $\Omega=\Omega(v) \subset \mathbb{R}^{2}$ with the following geometrical structure

$$
\Omega(v)=\left\{\left(x_{1}, x_{2}\right) \in \mathbb{R}^{2} \mid 0<x_{1}<v\left(x_{2}\right), 0<x_{2}<1\right\},
$$

where $\vee \in c^{0,1}([0,1]$ ) (i.e. $v$ Lipschitz-function); $\partial \Omega(v)=\Gamma_{1} U \Gamma(v)$ the boundary of $\Omega(v)$ with (see Figure 2.1)

$$
\begin{aligned}
& \Gamma_{1}=\partial \Omega(v)-\Gamma(v), \\
& \Gamma(v)=\left\{x \in \mathbb{R}^{2} \mid x_{1}=v\left(x_{2}\right), 0<x_{2}<1\right\} .
\end{aligned}
$$

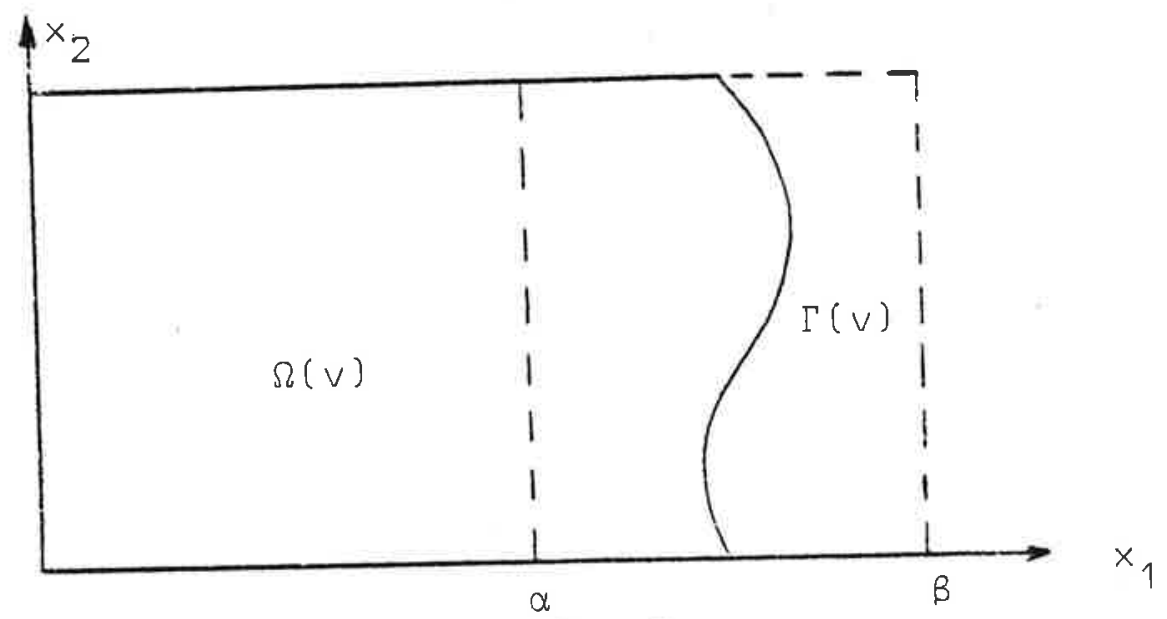

Figure 2.1. Domain $\Omega(v)$ with moving boundary $\Gamma(v)$

In this case the optimal shape design problem reduces to finding boundary $\Gamma(v)$ under certain criteria. Indeed, we can formulate our problem as follows:

Problem (P). (Optimal shape design) Find $w \in U_{\text {ad }}$ such that

$$
J(w)=\min _{v \in U_{\text {ad }}} J(v),
$$

where

$$
J(v)=\int_{\Omega(v)}\left|y(v)-z_{d}\right|^{2} d x
$$


$z_{d} \in L^{2}(\tilde{\Omega})$ a given function $(\tilde{\Omega}:=(0, \tilde{\beta}) \times(0,1), \tilde{\beta}>\beta)$ and $u_{\text {ad }}$ is a set of admissible controls,

$$
\begin{aligned}
& u_{a d}=\left\{v \in c^{0,1}([0,1]) \mid 0<\alpha \leq v\left(x_{2}\right) \leq \beta,\right. \\
& \left.\left|\frac{d v}{d x_{2}}\right| \leq c_{1}, \int_{0}^{1} v\left(x_{2}\right) d x_{2}=c_{2}\right\},
\end{aligned}
$$

$\alpha, \beta, c_{1}$ and $c_{2}$ are given positive constants. Function $y=y(v)$ is the solution of the unilateral boundary value problem: Find $y=y(v) \in K(\Omega(v))$ such that

$$
(\operatorname{grad} y, \operatorname{grad}(\xi-y))_{0, \Omega(v)} \geq(f,(\xi-y))_{0, \Omega(v)}
$$

for all $\xi \in K(\Omega(v))$. Here $f \in L^{2}(\tilde{\Omega})$ is a given function and

$$
\begin{aligned}
& V(\Omega(v))=\left\{\xi \in H^{1}(\Omega(v)) \mid \xi=0 \text { on } \Gamma_{1}\right\}, \\
& K(\Omega(v))=\{\xi \in V(\Omega(v)) \mid \xi \geq 0 \text { on } \Gamma(v)\},
\end{aligned}
$$

$(\ldots)_{0, \Omega(v)}$ denotes the scalar product in $L^{2}(\Omega(v))$.

In [15] the following fundamental result is proved for the solvability of Problem (P):

\section{Theorem 2.1. Problem (P) has at least one solution.}

In practice Problem ( $P$ ) has to be solved approximately. The unknown part of the boundary is sought among piecewise linear arcs and the state problem is solved by finite element method in polygonal domain $\Omega\left(v_{h}\right)$. In $\Omega\left(v_{h}\right)$ a moving finite element grid is constructed. Another possibility would be the transformation technique: the problem is transformed into an equivalent one with the state problem defined on a unit square domain and then finite elements on a uniform mesh are employed.

Anyhow, in both cases the main trouble is that the mapping $v \mapsto y(v)$ is not differentiable. As, moreover, the cost functional $v \rightarrow J(v)$ is not convex we are led to difficult optimization 
problem. To avoid the first difficulty in [10] another approach for (2.4) is utilized. Instead of (2.4) a family of penalized problems is considered: Find $y_{\varepsilon}=y_{\varepsilon}(v) \in V(\Omega(v))$ such that

$$
\begin{aligned}
& \left(\operatorname{grad} y_{\varepsilon}, \operatorname{grad} \xi\right)_{0, \Omega(v)}+\frac{1}{\varepsilon}\left(P\left(y_{\varepsilon}\right), \xi\right)_{v} \\
& =(f, \xi)_{0, \Omega(v)} \text { for all } \xi \in V(\Omega(v)) \text {, }
\end{aligned}
$$

where $P$ denotes the penalty mapping

$$
\left(P\left(y_{\varepsilon}\right), \xi\right)_{v}:=-\int_{0}^{1} y_{\varepsilon}^{-}\left(v\left(x_{2}\right), x_{2}\right) \xi\left(v\left(x_{2}\right), x_{2}\right) d x_{2},
$$

$y_{\varepsilon}^{-}=\left(\left|y_{\varepsilon}\right|-y_{\varepsilon}\right) / 2$ is the negative part of $y_{\varepsilon}$ and $\varepsilon>0$ is a penalty factor.

The regularized optimal shape design problem reads:

Problem (P) $\varepsilon$. Find $w_{\varepsilon} \in u_{\text {ad }}$ such that

$(2.6)$

$$
J\left(w_{\varepsilon}\right)=\min _{v \in U_{\text {ad }}} J(v)
$$

where

$$
J(v)=\int_{\Omega(v)}\left|y_{\varepsilon}(v)-z_{d}\right|^{2} d x
$$

and $y_{\varepsilon}$ is the solution of $(2.5)$.

The solvability of Problem $(P)_{\varepsilon}$ is proved in [10]:

Theorem 2.2. For any $\varepsilon>0$ Problem (P) ${ }^{(P}$ has at least one solution.

According to Theorem 2.2 there exists for any $\varepsilon_{k} \rightarrow 0+$ at least one optimal solution of Problem (P) $\varepsilon_{k}$, which will be denoted by $w_{k}$ and the corresponding state by ${ }^{\varepsilon_{k}} y_{k}\left(w_{k}\right)$. In [10] it is also proved that some solutions of Problem $(P) \varepsilon_{k}$ are close to a solution of Problem (P). Indeed, it holds ${ }^{k}$ (see [10], Theorem 4.1): 
Theorem 2.3. There exist a subsequence $\left\{w_{k_{j}}, y_{k_{j}}\left(w_{k_{j}}\right)\right\}$ of $\left\{w_{k}, y_{k}\left(w_{k}\right)\right\}$ and elements $w \in u_{\text {ad }}$, $y(w) \in K(\Omega(w))$ such that

$$
\begin{aligned}
& w_{k_{j}} \rightarrow w \text { (uniformly) } \underline{\text { in }}[0,1] \text { for } j \rightarrow \infty \\
& y_{k_{j}}\left(w_{k_{j}}\right)-y(w) \quad \text { (weakly) in } H^{1}\left(G_{m}(w)\right) \text { for } j \rightarrow \infty,
\end{aligned}
$$

and for any $\mathrm{m}$, where

$$
G_{m}(w)=\left\{\left(x_{1}, x_{2}\right) \in \mathbb{R}^{2} \mid 0<x_{1}<w\left(x_{2}\right)-1 / m, 0<x_{2}<1\right\},
$$

and where $w$ is the solution of Problem (P) and $y(w)$ is the corresponding state, solving the unilateral boundary value problem $(2.4)$ in $\Omega(w)$.

3. Finite element approximation dF the PENALIZED PROBLEM

According to Theorem 2.3 solutions of Problem ( $P$ ) and Problem ${ }^{(P)} \varepsilon_{\varepsilon}$ are in certain sense close together. It can be shown that similar result holds, if the penalized problem ( $P$ ) $\varepsilon$ is replaced by a family of finite element approximations. This fact will be utilized in discretization.

Let $0 \equiv a_{0}<a_{1}<\ldots<a_{N} \equiv 1$ be a partition of [0, 1 1 , $a_{i}-a_{i-1} \leq h$, and let

$$
u_{a d}^{h}=\left\{v_{h} \in c([0,1])\left|v_{h}\right|_{\left[a_{i-1}, a_{i}\right]} \in P_{1}\left(\left[a_{i-1}, a_{i}\right]\right)\right\} n u_{a d},
$$

where $P_{1}$ denotes the set of all linear functions.

For any $v_{h} \in u_{\text {ad }}$ we define

$$
\Omega\left(v_{h}\right)=\left\{x \in \mathbb{R}^{2} \mid x_{1} \in\left(0, v_{h}\left(x_{2}\right)\right), x_{2} \in(0,1)\right\},
$$


i.e. the variable part of the boundary $\Gamma_{2}(v)$ is now approximated by piecewise linear arc $F_{2}\left(v_{h}\right)$. By $T_{h}\left(v_{h}\right), v_{h} \in u_{a d}^{h}$, we denote the triangulation of $\Omega\left(v_{h}\right)$ such that the whole segment $I_{i}=\left\{\left(x_{1}, x_{2}\right) \mid x_{1}=v_{h}\left(x_{2}\right), x_{2} \in\left[a_{i-1}, a_{i}\right]\right\}$ is the whole side of a triangle $T_{i} \in T_{h}\left(v_{h}\right)$ and satisfying usual requirements, concerning the mutual position of two triangles, belonging to $T_{h}\left(v_{h}\right)$. Moreover, we shall assume such families of $\left\{T_{h}\left(v_{h}\right)\right\}, h \rightarrow 0+a n l y$, which are regular uniformly with respect to $v_{h} \in u_{a d}^{h}, i . e$. there exists $\alpha_{0}>0$ independently on $h>0$ and $v_{h} \in u_{a d}^{h}$ such that all interior angles of all triangles belonging to $T_{h}\left(v_{h}\right)$ are greater or equal to $\alpha_{0}$ (for practical applications some other technical restrictions will be added (see chapter 4.1)).

Finally, the symbol $\Omega_{h}\left(v_{h}\right)$ will denote the set $\Omega\left(v_{h}\right)$ with a given triangulation $T_{h}\left(v_{h}\right)$; we also use abbreviation $\Omega_{h}$ for $\Omega_{h}\left(v_{h}\right)$.

To any $T_{h}\left(v_{h}\right)$ a finite dimensional space $V_{h}\left(\Omega_{h}\left(V_{h_{1}}\right)\right.$ will be assoirated:

$$
\begin{aligned}
& v_{h}\left(\Omega_{h}\left(v_{h}\right)\right)=\left\{v_{h} \in C\left(\overline{\Omega_{h}\left(v_{h}\right)}\right)\left|v_{h}\right|_{T} \in P_{1}(T),\right. \\
& \left.\forall T \in T_{h}\left(v_{h}\right), v_{h}=0 \text { on } \Gamma_{1}=\partial \Omega_{h}\left(v_{h}\right)-\Gamma_{2}\left(v_{h}\right)\right\} .
\end{aligned}
$$

The approximation of the penalized optimal shape design problem is now defined as follows:

Problem (P) hind $w_{h} \in u_{a d}^{h}$ such that

$$
J_{h}\left(w_{h}\right)=\min _{v_{h} \in u_{\text {ad }}^{h}} J_{h}\left(v_{h}\right),
$$

where

$$
J_{h}\left(v_{h}\right)=\int_{\Omega_{h}\left(v_{h}\right)}\left|y_{h}\left(v_{h}\right)-z_{d}\right|^{2} d x
$$

and $y_{h}=y_{h}\left(v_{h}\right) \in v_{h}\left(\Omega_{h}\left(v_{h}\right)\right)$ is a solution of the nonlinear elliptic boundary value problem 
$(3.2)$

$$
\begin{aligned}
& \left(\text { grad } y_{h}, \operatorname{grad} \xi_{h}\right) 0, \Omega_{h}\left(v_{h}\right)+\frac{1}{\varepsilon(h)}\left(P\left(y_{h}\right), \xi_{h}\right) v_{h} \\
= & \left(f, \xi_{h}\right) 0, \Omega_{h}\left(v_{h}\right) \text { for all } \xi_{h} \in v_{h}\left(\Omega_{h}\left(v_{h}\right)\right) .
\end{aligned}
$$

The penalty operator is defined like in the continuous case and the penalty parameter $\varepsilon=\varepsilon(h)$ is such that $\varepsilon(h) \rightarrow 0+$ for $h \rightarrow 0+$.

Using classical compactness arguments one can prove

Theorem 3.1. Far any $h \in(0,1)$ there exists a solution $w_{h} \in u_{\text {ad }}^{h}$ of problem $(P)_{h}$.

The main result of this section is

Theorem 3.2. Let $w_{h} \in u_{\text {ad }}^{h}$ be a solution of Problem (P) $h$ and $y_{h}\left(w_{h}\right)$ the corresponding solution of the state equation (3.2). Then there exist a subsequence $\left\{w_{h}\right\} \subset\left\{w_{h}\right\}$, an element $w \in u_{\text {ad }}$ and $y(w) \in K(\Omega(w))$ such that

$$
\begin{aligned}
& w_{h_{j}} \rightarrow w(\underline{\text { uniformly }}) \text { in }[0,1], h_{j} \rightarrow 0+ \\
& y_{h_{j}}\left(w_{h_{j}}\right)+y(w) \underline{\text { in }} H^{1}\left(G_{m}(w)\right), h_{j} \rightarrow 0+
\end{aligned}
$$

for any $\mathrm{m}$, where $w$ is a solution of Problem (P) and $y(w)$ is a solution of the corresponding state inequality (2.4) in $\Omega(w)$.

For the proof of this theorem we need the following auxiliary result:

Lemma 3.3. Let $v_{h} \in u_{a d}^{h}$ be such that $v_{h} \rightarrow v^{\rightarrow}$ (uniformly) in [0,1]. Let $y_{h}=y_{h}\left(v_{h}\right)$ be a solution of (3.2) on domain $\Omega_{h}\left(v_{h}\right)$. Then there exists a subsequence $\left\{y_{h}\left(v_{h}\right)\right\} \subset \overline{\left\{y_{h}\left(v_{h}\right)\right\}}$ 


$$
y_{h_{j}}\left(v_{h}\right)+y(v) \text { in } H^{1}\left(G_{m}(v)\right)
$$

for $j \rightarrow \infty$ and for any natural $m$, where $y(v) \in K(\Omega(v))$ is the solution of variational inequality $(2.4)$ and $G_{m}(v)$ is defined analogously to $(2.7)$.

Proof. To simplify notations we shall write $\Omega_{h}$ and $y_{h}$ instead of $\Omega_{h}\left(v_{h}\right)$ and $y_{h}\left(v_{h}\right)$, when no confusion arises. The proof is similar to the one given for the continuous case [10] and it will be given in several steps.

1! First we prove that there exists $y=y(v) \in H^{1}(\Omega(v))$ and $\left\{y_{h}\left(v_{h}\right)\right\} \subset\left\{y_{h}\left(v_{h}\right)\right\}$ such that

$$
y_{h_{j}}\left(v_{h_{j}}\right)-y(v) \text { in } H^{1}\left(G_{m}(v)\right) \text { for any } m \text {. }
$$

The sequence $\left\{\left\|y_{h}\right\|_{1, \Omega_{h}}\right\}$ is bounded. Indeed,from (3.1) it
follows that

$$
\begin{aligned}
\| \text { grad } y_{h} \|_{0, \Omega_{h}}^{2} & \leq\left(\operatorname{grad} y_{h}, \text { grad } y_{h}\right)_{0, \Omega_{h}}+\frac{1}{\varepsilon(h)}\left(P\left(y_{h}\right), y_{h}\right) v_{h} \\
& =\left(f, y_{h}\right)_{0, \Omega_{h}} .
\end{aligned}
$$

From this, Poincare's generalized inequality and from the fact $0<\alpha \leq v_{h}\left(x_{2}\right) \leq \beta$ it follows that there exists a canstant $c>0$ independent of $h$ such that

$$
c\left\|y_{h}\right\|_{1, \Omega_{h}}^{2} \leq\|f\|_{0, \Omega_{h}}\left\|y_{h}\right\|_{0, \Omega_{h}} . .^{1)}
$$

1) In the sequel c denotes a generic constant, which doesn't depend on h. 
Hence

$(3.5)$

$$
\left\|y_{h}\right\|_{1, \Omega_{h}} \leq c \quad \text { for any } h .
$$

Let $m$ be fixed.

Then there exists $h_{0}=h_{0}(m)$ such that $\overline{G_{m}(v)} \subset \Omega_{h}$ for all $h \leq h_{0}$. Consequently by $(3.5)$

$$
\left\|y_{h}\right\|_{1, G_{m}(v)} \leq\left\|y_{h}\right\|_{1, \Omega_{h}} \leq c \quad \text { for all h } \leq h_{0} \text {. }
$$

As $H^{1}\left(G_{m}(v)\right)$ is a Hilbert space, there exists a $y^{(m)} \in H^{1}\left(G_{m}(v)\right)$ and a subsequence $\left\{y_{h}(m)\right\} \subset\left\{y_{h}\right\}$ such that

$$
y_{h}(m)-y^{(m)} \text { in } H^{1}\left(G_{m}(v)\right)
$$

Proceeding in the same way on $G_{m+1}(v)$ with $\{y(m)\}$ one can choose $\left\{y_{h}^{(m+1)}\right\} \subset\left\{y_{h}^{(m)}\right\}$ such, that

$$
y_{h}(m+1)-y^{(m+1)} \text { in } H^{1}\left(G_{m+1}(v)\right)
$$

and $y^{(m+1)}=0$ on $\partial G_{m+1}(v)-I_{m}(v)$, where

$$
I_{m}(v)=\left\{\left(x_{1}, x_{2}\right) \in \mathbb{R}^{2} \mid x_{1}=v\left(x_{2}\right)-1 / m, \quad x_{2} \in[0,1]\right\} .
$$

$$
\begin{array}{cl}
\text { Moreover } y^{(m+1)}=y^{(m)} & \text { on } G_{m} \text {. Setting } \\
y=y^{(m)} & \text { on } G_{m}(v) \text { for all } m
\end{array}
$$

we obtain $y \equiv y(v) \in H^{1}(\Omega(v)), y=0$ on $\partial \Omega(v) \backslash \Gamma(v)$. A diagonal sequence, constructed from $\left\{_{\mathrm{h}}(\mathrm{m})\right\}$ has the 
2: Let us prove that y $\in K(\Omega(v))$

For any $\xi \in H_{0}^{1}\left(\Omega_{\widetilde{\beta}}\right) \cap H^{2}\left(\Omega_{\widetilde{\beta}}\right)$ we define $\xi_{h_{j}}=\pi_{h_{j}}\left(\left.\xi\right|_{\Omega_{h}}\right)$, where $\pi_{h_{j}}$ denotes a mapping, which associates to any $\xi \in H^{2}\left(\Omega_{\tilde{B}}\right)$ its piecewise linear interpolate from $V_{h_{j}}\left(\Omega_{h_{j}}\right)$ ( $h_{j}$ is filter of indices for which (3.3) holds). Substituting $\xi_{h_{j}}$ into (3.2), we get

$\left(\operatorname{grad} y_{h_{j}}, \operatorname{grad} \xi_{h_{j}}\right) 0, \Omega_{h_{j}}+\frac{1}{\varepsilon\left(h_{j}\right)}\left(P\left(y_{h_{j}}\right), \xi_{h_{j}}\right) v_{h_{j}}=\left(f, \xi_{h_{j}}\right) 0, \Omega_{h_{j}}$.

From this follows

$(3.7)$

$$
\begin{aligned}
0 & \leq\left|\left(P\left(y_{h_{j}}\right), \xi_{h_{j}}\right) v_{h_{j}}\right| \\
& \leq \varepsilon\left(h_{j}\right)\left\{\|f\|_{0, \Omega_{\widetilde{\beta}}}+\left\|\operatorname{grad} y_{h_{j}}\right\|_{0, \Omega_{h}}\right\}\left\|\xi_{h_{j}}\right\| \dot{I}_{h_{j}, \Omega_{h_{j}}}
\end{aligned}
$$

By utilizing the approximating property of linear interpolate we find that

$$
\left\|\xi_{h_{j}}-\xi\right\|_{1, \Omega_{h_{j}}} \leq c h_{j}|\xi|_{2, \Omega_{h_{j}}} \leq c h_{j}|\xi|_{2, \Omega_{\tilde{\beta}}} .
$$

Consequently

$$
\left\|\xi_{h_{j}}\right\|_{1, \Omega_{h_{j}}} \leq\left\|\xi_{h_{j}}-\xi\right\|_{1, \Omega_{h_{j}}}+\|\xi\|_{1, \Omega_{h_{j}}} \leq c .
$$

Taking into account this, (3.5) and (3.7) we have

$$
\lim _{h_{j \rightarrow 0+}}\left(P\left(y_{h_{j}}\right), \xi_{h_{j}}\right) v_{h_{j}}=0 .
$$


On the other hand,

$(3.9)$

$$
\begin{aligned}
& \left|(P(y), \xi)_{v}-\left(P\left(y_{h_{j}}\right), \xi_{h_{j}}\right)_{v_{h}}\right| \\
& \leq\left|(P(y), \xi)_{v}-\left(P\left(y_{h_{j}}\right), \xi\right)_{v_{h}}\right|+\left|\left(P\left(y_{h_{j}}\right), \xi-\xi_{h_{j}}\right) v_{h_{j}}\right| .
\end{aligned}
$$

The first term on the right hand side of (3.9) tends to zero, as follows from Lemma 3.2. in [10]. Let us estimate the second term:

$$
\begin{aligned}
& \left|\left(P\left(y_{h_{j}}\right), \xi-\xi_{h_{j}}\right) v_{h_{j}}\right|=\left|\int_{0}^{1} y_{h_{j}}^{-}\left(\xi\left(v_{h_{h}}\right)-\xi_{h_{j}}\left(v_{h_{j}}\right)\right) d x_{2}\right| \\
& \leq c\left\|y_{h_{j}}\right\|_{0, \Gamma\left(v_{h}\right)}\left\|\xi\left(v_{h_{j}}\right)-\xi_{h_{j}}\left(v_{h_{j}}\right)\right\|_{0, \Gamma\left(v_{h}\right)} \\
& \leq c\left\|y_{h_{j}}\right\|_{1, \Omega_{h_{j}}}\left\|\xi-\xi_{h_{j}}\right\|_{1, \Omega_{h_{j}}} \rightarrow 0,
\end{aligned}
$$

if $h_{j} \rightarrow 0^{+}$. In the last inequality we have used the fact that the norm of the trace mapping $y: V\left(\Omega\left(v_{h}\right)\right) \rightarrow L^{2}\left(\Gamma\left(v_{h}\right)\right)$ can be estimated uniformly with respect to h for sufficiently small h (in our situation this fact follows immediately from the proof of trace theorem, see [19], Th 2.1). Comparing (3.8) with (3.9) we see that

$$
\lim _{h_{j} \rightarrow 0^{+}}\left(P\left(y_{h_{j}}\right), \xi_{h_{j}}\right)_{v_{h_{j}}}=(P(y), \xi)_{v}=0
$$

for any $\xi \in H_{0}^{1}\left(\Omega_{\tilde{\beta}}\right) \cap H^{2}\left(\Omega_{\tilde{\beta}}\right)$, i.e. $y \geq 0$ on $\Gamma(v)$.

3! To establish the assertion of Lemma 3.3 ., it remains to verify that $y$ is a solution of variational inequality (2.4). Let $\xi \in K(\Omega(v))$. Then there exists a function $\psi \in H^{1}\left(\Omega_{\tilde{\beta}}\right)$ such
that

$$
\psi|\partial \Omega(v)=\xi| \partial \Omega(v)
$$


and $\psi \geq 0$ in $\Omega_{\beta}$. Setting

$$
n=\xi-\psi
$$

it is readily seen that $n \in H_{0}^{1}(\Omega(v))$ and therefore

$$
\eta=\lim _{i \rightarrow \infty} \eta_{i} \quad \text { in } H^{1}(\Omega(v))
$$

with $\eta_{i} \in D(\Omega(v))$. Similarly, one can prove the existence of $\psi_{i} \in C^{\infty}\left(\bar{\Omega}_{\widetilde{\beta}}\right) \quad \psi_{i} \geq 0$ in $\Omega_{\tilde{B}}$, such that

$$
\psi_{i} \rightarrow \psi \text { in } H^{1}\left(\Omega_{\widetilde{\beta}}\right)
$$

Let us define

$$
\xi_{i}= \begin{cases}\psi_{i}+n_{i} & \text { in } \Omega(v) \\ \psi_{i} & \text { in } \Omega_{\widetilde{\beta}}-\Omega(v) .\end{cases}
$$

Clearly $\xi_{i} \in C^{\infty}\left(\overline{\Omega_{\widetilde{B}}}\right)$,

$$
\xi_{i} \rightarrow \psi+\eta=\xi \quad \text { in } H^{1}(\Omega(v))
$$

and

$$
\xi_{i} \in K_{m}(\Omega(v))=\left\{z \in H^{1}\left(\Omega_{\widetilde{\beta}}\right) \mid z \geq 0 \text { in } \Omega_{\tilde{\beta}}-G_{m}(v)\right\}
$$

for all sufficiently large $i$. Let

$$
k_{h}\left(\Omega_{h}\left(v_{h}\right)\right)=\left\{\xi_{h} \in v_{h}\left(\Omega_{h}\right) \mid \xi_{h} \geq 0 \text { on } \Gamma\left(v_{h}\right)\right\}
$$

Then

$$
\xi_{i h}=\pi_{h}\left(\left.\xi_{i}\right|_{\Omega_{h}}\right) \in K_{h}\left(\Omega_{h}\right)
$$


if $i$ is large and $h$ is small. Let $h_{j}, j \rightarrow \infty$ be filter of indices, for which (3.3) holds.

Consider the problem (3.2) in which a function $\xi_{h}$ is replaced by $y_{h_{j}}-\xi_{i h_{j}}$. As $\xi_{i h_{j}} \in K_{h_{j}}\left(\Omega_{h_{j}}\right)$ and $P\left(\xi_{i h_{j}}\right)=0$, we obtain by utilizing the monotonity of $P$ that

(3.10) (grad $\left.y_{h_{j}}, \operatorname{grad} y_{h_{j}}-\operatorname{grad} \xi_{i h_{j}}\right)_{0, \Omega_{h_{j}}} \leq\left(f, y_{h_{j}}-\xi_{i h_{j}}\right) 0, \Omega_{h_{j}}$. Furthermore, it holds

$(3.11)$

$$
\begin{aligned}
& \left(\operatorname{grad} y_{h_{j}}, \operatorname{grad}\left(\xi_{i h_{j}}-y_{h_{j}}\right)\right)_{0, \Omega_{h_{j}}} \\
& =\left(\operatorname{grad} y_{h_{j}}, \operatorname{grad}\left(\xi_{i h_{j}}-y_{h_{j}}\right)\right)_{0, G_{m}}(v) \\
& +\left(\operatorname{grad} y_{h_{j}}, \operatorname{grad}\left(\xi_{i h_{j}}-y_{h_{j}}\right)\right)_{0, \Omega_{h_{j}}}-\Omega(v) \\
& +\left(\operatorname{grad} y_{h_{j}}, \operatorname{grad}\left(\xi_{i h_{j}}-y_{h_{j}}\right)\right){ }_{0},\left(\Omega(v)-G_{m}(v)\right) \cap \Omega_{h_{j}} \\
& \leq\left(\operatorname{grad} y_{h_{j}}, \operatorname{grad}\left(\xi_{i h_{j}}-y_{h_{j}}\right)\right) 0, G_{m}(v) \\
& +\left(\operatorname{grad} y_{h_{j}}, \operatorname{grad} \xi_{i h_{j}}{ }^{\prime} 0, \Omega_{h_{j}}-\Omega(v)\right. \\
& +\left(\operatorname{grad} y_{h_{j}}, \operatorname{grad} \xi_{i h_{j}} 0,\left(\Omega(v)-G_{m}(v)\right) \cap \Omega_{h_{j}} .\right.
\end{aligned}
$$

As

$$
y_{h_{j}} \rightarrow y \quad \text { in } H^{1}\left(G_{m}(v)\right)
$$

and

$$
\xi_{i h_{j}} \rightarrow \xi_{i} \text { in } H^{1}\left(G_{m}(v)\right)
$$


then

$(3.12)$

$$
\begin{aligned}
& \quad \lim _{h_{j} \rightarrow 0^{+}} \sup \left(\operatorname{grad} y_{h_{j}}, \operatorname{grad}\left(\xi_{i h_{j}}-y_{h_{j}}\right)\right)_{0, G_{m}}(v) \\
& \leq\left(\operatorname{grad} y, \operatorname{grad}\left(\xi_{i}-y\right)\right)_{C, G_{m}}(v) .
\end{aligned}
$$

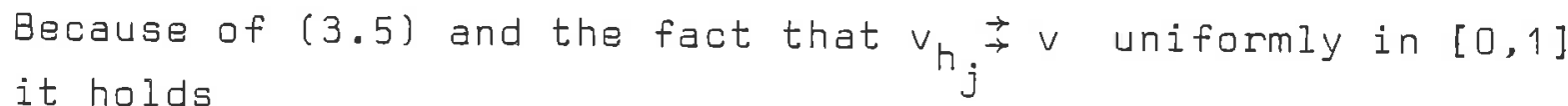

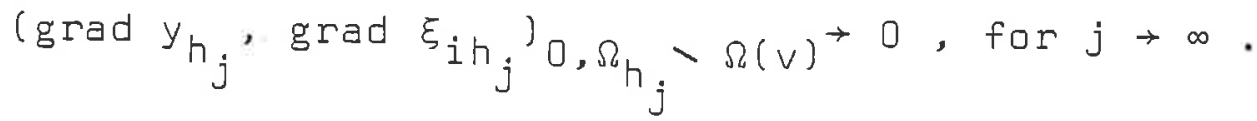

As

$$
\begin{aligned}
& \left|\left(\operatorname{grad} y_{h_{j}}, \operatorname{grad}\left(\xi_{i h_{j}}-\xi_{i}\right)\right) 0,\left(\Omega(v) \backslash G_{m}(v)\right) \cap \Omega_{h_{j}}\right| \\
\leq & c\left\|\xi_{i h_{j}}-\xi_{i}\right\|_{1, \Omega_{h_{j}}} \leq c h_{j}\left|\xi_{i}\right|_{2, \Omega(v)} \rightarrow 0 \text { for } j \rightarrow \infty
\end{aligned}
$$

we can conclude that

. $\lim _{j \rightarrow \infty} \sup \left(\right.$ grad $y_{h_{j}}$, grad $\left.\xi_{i h_{j}}\right),\left(\Omega(v)-G_{m}(v)\right) \cap \Omega_{h_{j}}$

$\leq \lim _{j \rightarrow \infty} \sup \left(\operatorname{grad} y_{h_{j}}, \operatorname{grad} \xi_{i}\right) 0,\left(\Omega(v)-G_{m}(v)\right) \cap \Omega_{h}$

$+\lim _{j \rightarrow \infty} \sup \left(\operatorname{grad} y_{h_{j}}, \operatorname{grad}\left(\xi_{i h_{j}}-\xi_{i}\right)\right) O,\left(\Omega(v)-G_{m}(v)\right) \cap \Omega_{h_{j}}$.

From this, (3.11), (3.12) and (3.13) we obtain that

$$
\begin{aligned}
& \lim _{j \rightarrow \infty} \sup \left(\operatorname{grad} y_{h_{j}}, \operatorname{grad}\left(\xi_{i h_{j}}-y_{h_{j}}\right)\right)_{0, \Omega_{h_{j}}} \\
\leq & \left(\operatorname{grad} y(v), \operatorname{grad}\left(\xi_{i}-y(v)\right)\right)_{0, G_{m}(v)}{ }^{+} c\left\|\xi_{i}\right\|_{1, \Omega(v) \backslash G_{m}(v) .}
\end{aligned}
$$


Finally, we estimate the linear term:

$$
\begin{aligned}
& \left(f, \xi_{i h_{j}}-y_{h_{j}}\right) 0, \Omega_{h_{j}}=\left(f, \xi_{i h_{j}}-y_{h_{j}}\right), G_{m}(v) \\
& +\left(f, \xi_{i h_{j}}-y_{h_{j}}\right) \Omega_{, \Omega_{h_{j}}} \Omega(v)+\left(f, \xi_{i h_{j}}-y_{h_{j}}\right),\left(\Omega(v) \backslash G_{m}(v)\right) \cap \Omega_{h_{j}} .
\end{aligned}
$$

Hence,

$$
\begin{aligned}
& \lim _{j \rightarrow \infty} \inf \left(f, \xi_{i h_{j}}-y_{h_{j}}\right)_{0, \Omega_{h}} \\
& \geq\left(f, \xi_{i}-y\right)_{0, G_{m}(v)}-c\left\{\|f\|_{0, \Omega(v)} G_{m}(v)+\left\|\xi_{i}\right\|_{1, \Omega(v) \backslash G_{m}(v)}\right\} .
\end{aligned}
$$

By summing $(3.10)-(3.15)$ we have

$$
\begin{aligned}
& \left(\operatorname{grad} y(v), \operatorname{grad}\left(\xi_{i}-y(v)\right)\right)_{0, G_{m}(v)}+c\left\|\xi_{i}\right\|_{1, \Omega(v)} G_{m}(v) \\
\geq & \left(f, \xi_{i}-y(v)\right)_{0, G_{m}(v)}-c\left\{\|f\|_{0, \Omega(v) \backslash G_{m}(v)}+\left\|\xi_{i}\right\|_{1, \Omega(v) \backslash G_{m}(v)}\right\} .
\end{aligned}
$$

Letting $m \rightarrow \infty$ we have

$$
\left(\operatorname{grad} y(v), \operatorname{grad}\left(\xi_{i}-y(v)\right)\right)_{0, \Omega(v)} \geq\left(f, \xi_{i}-y(v)\right)_{0, \Omega(v)} \cdot
$$

Thence, if $i \rightarrow \infty$ we have

$$
(\operatorname{grad} y(v), \operatorname{grad}(\xi-y(v)))_{0, \Omega(v)} \geq(f, \xi-y(v))_{0, \Omega(v)}
$$

for any $\xi \in k(\Omega(v))$. This completes the proof of Lemma 3.3. 
Now we are able to prove Theorem 3.2.

Proof of Theorem 3.2. Let $\left\{w_{h}\right\}, h \rightarrow 0+, w_{h} \in u_{\text {ad }}^{h}$ are solutions of Problem (P) $h$. As $u_{\text {ad }}^{h} \subset U_{\text {ad }}$ for all h and $u_{\text {ad }}$ is compact in $c^{0}$-topology, there exists a subsequence $\left\{w_{h}\right\} \subset\left\{w_{h}\right\}$ and $w \in u_{\text {ad }}$ such that

$$
w_{h_{j}} \rightarrow w \in u_{\text {ad }} \text { uniformly in }[0,1] \text {. }
$$

Let $\left\{y_{h_{j}}\left(w_{h}\right)\right\}$ be corresponding solutions of state problem (3.2). In accordance with Lemma 3.3, there exists a subsequence $\left\{y_{h}\left(w_{h}\right)\right\}$ (we use the same notation) and an element $y(w) \in K(w)$ such that

$$
y_{h_{j}}\left(w_{h}\right) \rightarrow y(w) \quad \text { in } H^{1}\left(G_{m}(w)\right)
$$

for any $m$, and moreover, $y(w)$ solves the state inequality $(2.4)$.

We now show that $w$ solves Problem $(P)$. Let $v \in u_{\text {ad }}$ be an arbitrary function. Then there exists $\bar{v}_{h} \in u_{a d}^{h}$ such that

$$
\bar{v}_{h} \rightarrow v \quad \text { uniformly in }[0,1]
$$

(see [3]). By definition of Problem (P)h one has:

$$
I_{h}\left(w_{h}\right) \leq J_{h}\left(\bar{v}_{h}\right) \text { for all } v_{h} \in u_{\text {ad }}^{h} \text {. }
$$

Let us write

$$
\begin{aligned}
J_{h_{j}}\left(w_{h_{j}}\right) & =\left\|y_{h_{j}}\left(w_{h_{j}}\right)-z_{d}\right\|_{0, G_{m}(w)}^{2}+\left\|v_{h_{j}}\left(w_{h_{j}}\right)-z_{d}\right\|_{0, \Omega_{h_{j}}}^{2}\left(w_{h_{j}}\right) \backslash G_{m}(w) \\
& \geq\left\|y_{h_{j}}\left(w_{h_{j}}\right)-z_{d}\right\|_{0, G_{m}}^{2}(w)
\end{aligned}
$$


so that

$(3.17)$

$$
\begin{aligned}
& \left\|y_{h_{j}}\left(w_{h_{j}}\right)-z_{d}\right\|_{0, G_{m}}^{2}(w) \leq J_{h_{j}}\left(w_{h_{j}}\right) \leq J_{h_{j}}\left(\bar{v}_{h_{j}}\right) \\
= & \left\|y_{h_{j}}\left(v_{h_{j}}\right)-z_{d}\right\|_{0, G_{m}}^{2}(v)+\left\|y_{h_{j}}\left(v_{h_{j}}\right)-z_{d}\right\|_{0, \Omega_{h_{j}}}^{2}\left(v_{h_{j}}\right)-G_{m}(v) .
\end{aligned}
$$

Here $y_{h_{j}}\left(\bar{v}_{h_{j}}\right)$ denotes the solution of $(3.2)$ on $\Omega_{h_{j}}\left(\bar{v}_{h_{j}}\right)$.

Now the indices are chosen in such a way that both $\left\{y_{h}\left(w_{h}\right)\right\}$ as well as $\left\{y_{h_{j}}\left(\bar{v}_{h_{j}}\right)\right\}$ tend weakly to $y(w)$ and to $y(v)$ in $H^{1}\left(G_{m}(w)\right)$ and $H^{1}\left(G_{m}(v)\right)$, respectively. Applying Rellich's theorem to $(3.17)$ we get

$$
\begin{aligned}
& \left\|y(w)-z_{d}\right\|_{0, G_{m}(w)}^{2} \\
& \leq\left\|y(v)-z_{d}\right\|_{0, G_{m}(v)}^{2}+\underset{j \rightarrow \infty}{\lim \sup }\left\|y_{h_{j}}\left(\bar{v}_{h_{j}}\right)-z_{d}\right\|_{0, \Omega_{h}}^{2}\left(\bar{v}_{h}\right) \backslash G_{j}(v) .
\end{aligned}
$$

Let us analyse the last term on the right hand side of (3.18). One can write

$$
\begin{aligned}
& \quad\left\|y_{h_{j}}\left(\bar{v}_{h_{j}}\right)-z_{d}\right\|_{0, \Omega_{h_{j}}}^{2}\left(\bar{v}_{h_{j}}\right)-G_{m}(v) \\
& \leq\left(\operatorname{meas}\left(\Omega_{h_{j}}\left(\bar{v}_{h_{j}}\right) \backslash G_{m}(v)\right)\right)^{1 / 2}\left\|y_{h_{j}}\left(\bar{v}_{h_{j}}\right)-z_{d}\right\|_{L}^{2}\left(\Omega_{h_{j}}\left(\bar{v}_{h_{j}}\right)\right)
\end{aligned}
$$

We define a mapping $F_{h}: \mathbb{R}^{2} \rightarrow \mathbb{R}^{2}$ by means of the relation

$$
F_{h}\left(\hat{x}_{1}, \hat{x}_{2}\right)=\left(\hat{x}_{1} v_{h}\left(\hat{x}_{2}\right), \hat{x}_{2}\right),
$$

$\left(\hat{x}_{1}, \hat{x}_{2}\right) \in \hat{\Omega}:=(0,1) \times(0,1)$. Then $\Omega_{h_{j}}\left(\bar{v}_{h_{j}}\right)=F_{h_{j}}(\hat{\Omega})$. 
Fubini's theorem and the continuity of the imbedding $H^{1}(\hat{\Omega}) \hookrightarrow L^{4}(\hat{\Omega})$ imply

$(3.20)$

$$
\begin{aligned}
& \left.\left\|y_{h_{j}}\left(\bar{v}_{h_{j}}\right)-z_{d}\right\|_{L^{4}}^{2} \Omega_{h_{j}}\left(\bar{v}_{h_{j}}\right)\right) \\
& \leq c\left\|\hat{y}_{h_{j}}-\hat{z}_{d_{j}}\right\|_{L}^{2}(\hat{\Omega}) \leq \hat{c}\left\|\hat{y}_{h_{j}}-\hat{z}_{d_{j}}\right\|_{1, \hat{\Omega}}^{2},
\end{aligned}
$$

when $\hat{y}_{h_{j}}=y_{h_{j}} \circ F_{h_{j}}, \hat{z}_{d_{j}}=z_{d} \circ F_{h_{j}}$ and $\hat{c}>0$ is independent of $h_{j}$. Applying Fubini's theorem once again as well as (3.5) we get

$$
\left\|\hat{y}_{h_{j}}-\hat{z}_{u_{j}}\right\|_{1, \hat{\Omega}}^{2} \leq c\left\|y_{h_{j}}-z_{d}\right\|_{1, \Omega_{h_{j}}^{2}}^{2} \leq c .
$$

This and (3.19) imply the following estimate:

$$
\begin{aligned}
& \lim _{j \rightarrow \infty} \sup \left\|y_{h j}\left(\bar{v}_{h_{j}}\right)-z_{d}\right\|_{0}^{2}, \Omega_{h_{j}}\left(v_{h_{j}}\right) \backslash G_{m}(v) \\
& \leq c\left\{\operatorname{meas}\left(\Omega(v) \backslash G_{m}(v)\right)\right\}^{1 / 2} .
\end{aligned}
$$

A combination of (3.18) and (3.21) yields

$$
\begin{aligned}
& \left\|y(w)-z_{d}\right\|_{0, G_{m}(v)}^{2} \\
\leq & \left\|y(v)-z_{d}\right\|_{0, G_{m}}^{2}(v)^{+c}\left\{\text { meas } \Omega(v)-G_{m}(v)\right\}^{1 / 2} .
\end{aligned}
$$

Passing to the limit with $m \rightarrow \infty$, we finally get

$$
J(w) \leq J(v) \quad \text { for all } v \in u_{\text {ad }} \text {. }
$$

Thence Theorem 3.2 is proved. 
4. NUMERICAL REALIZATION OF OPTIMAL SHAPE DESIGN PROBLEM

4.1 Construction of moving triangular grid

Let $\Omega_{h}\left(v_{h}\right)=\hat{\Omega}^{\prime} \cup \Omega_{h}^{r}\left(v_{h}\right)$, where $\hat{\Omega}^{\prime}=\left(0, \alpha^{\prime}\right) \times(0,1), \alpha^{\prime}<\alpha$, is a part of $\Omega_{h}\left(v_{h}\right)$, where the moving boundary $\Gamma_{2}\left(v_{h}\right)$ cannot penetrate and $\Omega_{h}^{\Gamma}\left(v_{h}\right)$ the rest of $\Omega_{h}\left(v_{h}\right)$ (see Figure 4.1).

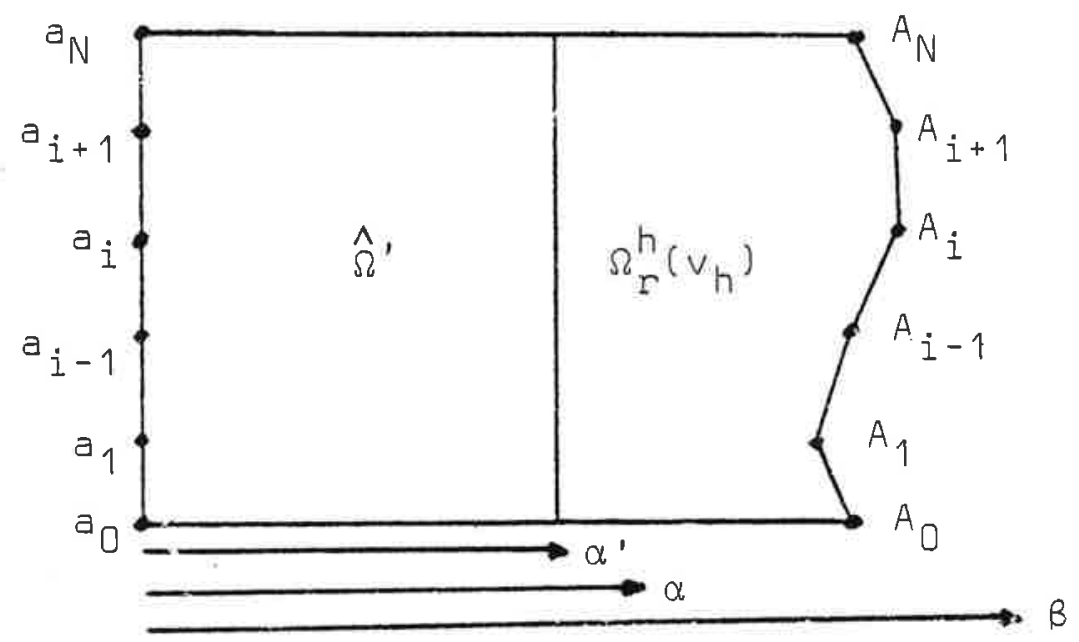

Figure 4.1. $\Omega_{h}\left(v_{h}\right)=\hat{\Omega}^{\prime} \cup \Omega_{h}^{r}\left(v_{h}\right)$.

Let $\hat{T}_{h}$ and $T_{h}^{\Gamma}\left(v_{h}\right)$ resp. De a triangulation of $\hat{\Omega}^{\prime}$ and of $\Omega_{h}^{\Gamma}\left(v_{h}\right)$ resp. $T_{h}^{\Gamma}\left(v_{h}\right)$ will be constructed my means of principle moving points

$(4.1)$

$$
A_{i}=\left(x_{1}^{i}, a_{i}\right), x_{1}^{i}=v_{h}\left(a_{i}\right), a_{i}=i h,
$$

further by means of associated moving points

$$
A_{i}^{j}=\left(\varphi_{i}^{j}\left(x_{1}^{i}\right), a_{i}\right)
$$

and of fixed points 


$$
\hat{A}_{i}=\left(\alpha^{\prime}, a_{i}\right)
$$

$i=0, \ldots, N, \quad j=1, \ldots, M, N=1 / h, \quad M=[\alpha / h]$.

It is readily seen that principal moving nodes and associate moving nodes may vary in $x_{1}$-direction only. The position of moving points is associated to principal moving points by means of function $\varphi_{i}^{j}$. If the dependence is supposed to be linear one may define

$$
\varphi_{i}^{j}\left(x_{1}^{i}\right)=\alpha^{\prime}+\frac{x_{1}^{i}-\alpha^{\prime}}{M} j, j=0, \ldots, M .
$$

Finally, $T_{h}^{\Gamma}\left(v_{h}\right)$ will be constructed by points $A_{i}, A_{i}^{j}$ and $\hat{A}_{i}$ such that triangulation of $\Omega_{h}^{r}\left(v_{h}\right)$ will be regular (see figure $4.2)$

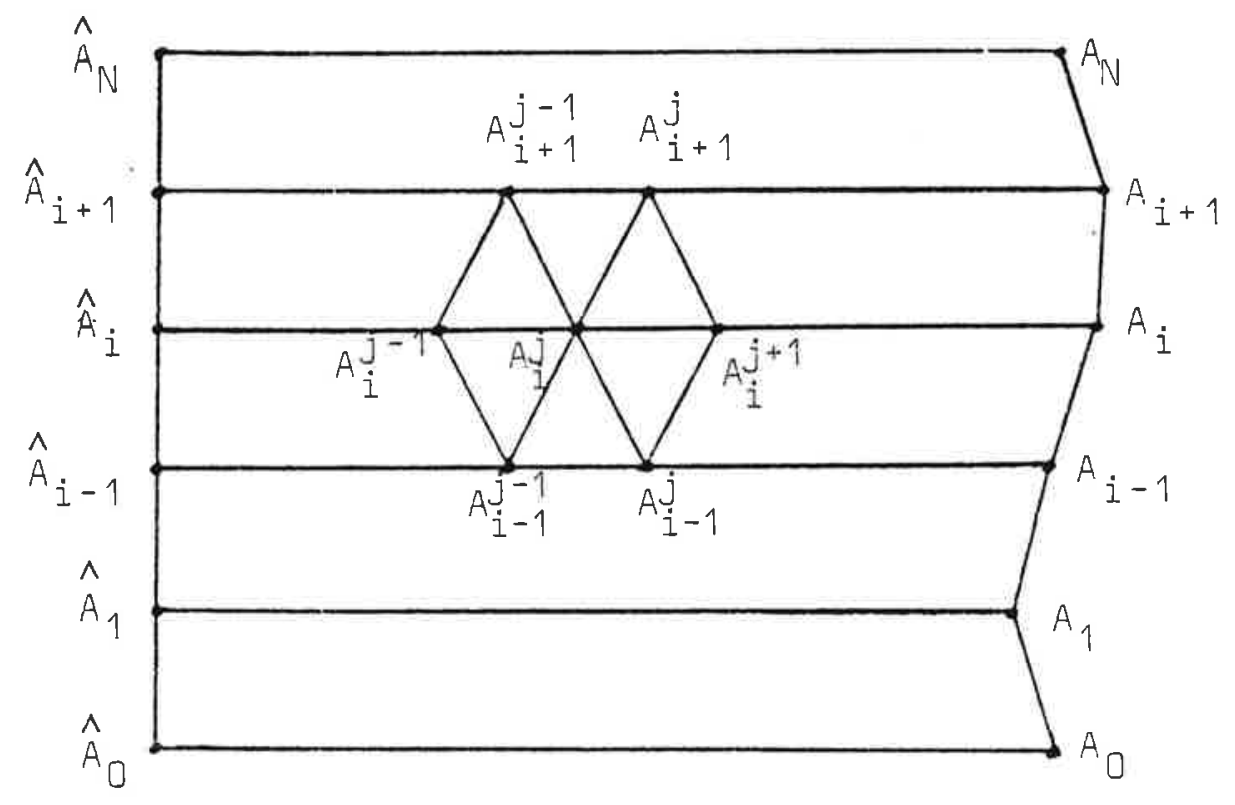

Figure 4.2. Triangulation of $\Omega_{h}^{\Gamma}\left(v_{h}\right)$.

Evidently $T_{h}\left(v_{h}\right)=\hat{T}_{h} \cup T_{h}^{r}\left(v_{h}\right)$ satisfy the assumptions desired in chapter 2 for regular triangulation of $\Omega\left(v_{h}\right)$. 
4.2 Algebraic formulation of discretized shape design problem

Taking into account the geometry of $\Omega_{h}\left(v_{h}\right)$ and piecewise linearity of $\Gamma\left(v_{h}\right)$, for finding optimal $\Gamma\left(v_{h}\right)$ it is enough to find the $x_{1}$-coordinates of the principal moving nodes (determining $\Gamma\left(v_{h}\right)$ ) such that $J_{h}$ is minimum. As for fixed $h$, the shape of $\Omega_{h}\left(v_{h}\right)$ and value of $y_{h}$ resp. depends on design variables $\left(x_{1}\right.$-coordinates of $\left.A_{i}\right)$

$$
x=\left(x_{1}^{0}, \ldots, x_{1}^{N}\right)
$$

we shall write $\Omega_{h}(x)$ and $y_{h}(x)$ resp. and instead of $\left(x_{1}^{0}, \ldots, x_{1}^{N}\right)$ we shall write simply $\left(x_{0}, \ldots, x_{N}\right)$.

For fixed $\Omega_{h}(X), y_{h}(X)$ can be obtained by solving the algebraic form of (3.2) for nodal displacements

$$
Q(x)=\left(a_{1}, \ldots, a_{p}, q_{p+1}, \ldots, q_{p-N-1}\right)^{\top}
$$

$\left(p=\right.$ number of interior nodes of $\left.T_{h}\left(v_{h}\right)\right)$ :

$$
A(X) Q(X)-\frac{1}{\varepsilon} D(Q(X))=F(X) .
$$

Here $A(X)$ is the global stiffness matrix, which presents in $V_{h}\left(\Omega_{h}(X)\right)$ the piecewise linear finite element discretization of $-\Delta, F(X)$ is the corresponding discrete right hand side; the dependence of these factors on design variables is emphasized here. Operator $D: \mathbb{R}^{K(h)} \rightarrow \mathbb{R}^{K(h)}, K(h)=\operatorname{dim} V_{h}\left(\Omega_{h}(X)\right)$, $K(h)=p+N-1$, is a nonlinear mapping corresponding to the second term of (3.2) with penalty operator $P\left(y_{\varepsilon}\right)=-\left(y_{\varepsilon}^{-}\right)^{2}$,

$$
D(Q)=\left(0, \ldots, 0, h\left(q_{p+1}^{-}\right)^{2}, \ldots, h\left(q_{p+N-1}^{-}\right)^{2}\right),
$$


where $q_{p+1}, \cdots, q_{p+N-1}$ are nodal displacements at principal moving nodes $\left(A_{1}, \ldots, A_{N-1}\right)$.

For the sake of smoothness, we have slightly modified the penalty operator from that of the previous chapters (this is allowed in the finite dimensional case, at least). The differentiability of the mapping $Q \rightarrow Q(Q)$ will be utilized in sensitivity analysis (chapter 4.3 ).

In order to express the cost functional $J_{h}$ by means of nodal displacements, we first find that it locally holds ffor the sake of simplicity we assume $z_{d} \equiv 0$ ):

$$
\begin{aligned}
& J_{h}^{T}:=\int_{T}\left(y_{h}(x)\right)^{2} d x \\
& =\frac{\operatorname{meas} T}{6}\left(q_{i}(x)^{2}+q_{j}(x)^{2}+q_{k}(x)^{2}\right. \\
& \left.+q_{i}(x) q_{j}(x)+q_{j}(x) q_{k}(x)+q_{i}(x) q_{k}(x)\right) .
\end{aligned}
$$

The dependence of the nodal displacements on design variables is emphasized here. We note that meas $T$ depends on $X$, if vertices belong to principal moving nodes or to associated moving nodes.

If we write $u_{\text {ad }}^{\text {h }}$ in the form

$$
\begin{aligned}
u_{a d}^{h}= & \left\{v_{h} \in C([0,1])\left|v_{h}\right|_{\left[a_{i-1}, a_{i}\right]} \in p_{1}, \alpha \leq v_{h} \leq \beta,\right. \\
& \left.\left|v_{h}(t)-v_{h}\left(t^{\prime}\right)\right| \leq c_{1}\left|t-t^{\prime}\right|, \int_{0}^{1} v_{h}(t) d t=c_{2}\right\}
\end{aligned}
$$

and take into account the local formula (4.5) we can formulate problem ( $P)_{h}$ for fixed $h$ and $\varepsilon(h)$ as the following nonlinear programming problem with linear constraints: 
(4.7) Minimize $\psi(X, Q(X))$

subject to constraints
(4.8)
$\alpha \leq x_{i} \leq \beta$
$i=0, \ldots, N$,
$(4.9)$
$c x \geq b$.

Here

$$
\psi(x, Q(X))=\sum_{i, j=1}^{K(h)} b_{j}(x) q_{i}(x) q_{j}(x)
$$

denotes the objective function obtained from $J_{h}\left(v_{h}\right)=\int_{\Omega_{h}(v)}\left(y_{h}\left(v_{h}\right)\right)^{2} d x$ by the local formula (4.5); (4.8), (4.9) correspond to constraints, given in the definition of $(4.6)$.

When the discretization of the continuous model has been done (by finite elements here), the backbone of a computer program for the numerical solution of the design problem is an optimization algorithm (quasi-Newton, conjugate gradient, projected gradient etc., as a rule a gradient algorithm). If the gradient with respect to design variables is not available, one must utilize nonsmooth aptimization algorithms (methods without derivatives, derivatives with finite differences or subgradient methods). The benefit of the penalty method over variational inequality formulation of the state problem is that it enables us to use gradient methods.

It turns out that the gradient with respect to the design variables (variation of the boundary) is an involved step. The next section is devoted to this single step. 
4.3 Computation of the gradient for the cost functional

By local formula $(4.5), \frac{\partial}{\partial x_{1}} \psi(X, Q(X)), 1=0, \ldots, N$, consists of terms $\frac{\partial}{\partial x_{1}}$ meas $T_{i}, T_{i} \in T_{h}^{T} r_{h}\left(v_{h}\right)$, and $\frac{\partial}{\partial x_{1}} q_{i}(X)$ :

(4.10) $\quad \frac{\partial}{\partial x_{1}} \psi(x, Q(x))=\sum_{i, j=1}^{k(h)}\left(\frac{\partial}{\partial x_{1}} b_{i j}(x)\right) q_{i}(x) q_{j}(x)$

$+\sum_{i, j=1}^{K(h)}\left[b_{i j}(x)\left(\frac{\partial}{\partial x_{l}} q_{i}(x) q_{j}(x)+q_{i}(x) \frac{\partial}{\partial x_{l}} q_{j}(x)\right)\right]$.

As $\frac{\partial}{\partial x_{1}}$ meas $T_{i}$ is evident to compute, the only problem in (4.10) is to find $\frac{\partial}{\partial x_{l}} q_{i}$, i.e. derivatives of nodal displacements with respect to design variables $x_{1}, 1=0, \ldots, N$. We are led to question: how the nodal displacements are varying when the boundary $\Gamma\left(v_{h}\right)$ is varying? We shall make use of the implicit function theorem.

Let

$(4.11)$

$\Phi(Q, X)=A(X) Q-\frac{1}{\varepsilon} D(Q)-F(X)$

and let $x$ be fixed (i.e. $\Gamma\left(v_{h}\right)$ is fixed). Then the problem: Find $Q=Q(X)$ such that

$$
\Phi(Q, X)=0
$$

is equivalent to (4.3). Now

$$
\frac{\partial}{\partial Q} \Phi \frac{\partial}{\partial x_{1}} Q+\frac{\partial}{\partial x_{1}} \Phi=0, \quad 1=0, \ldots, N
$$

is by (4.3) equivalent to equation 
$(4.13)$

$$
\left(A(X)-\frac{1}{\varepsilon} Q^{\prime}(Q)\right) \frac{\partial}{\partial x_{1}} Q=\frac{\partial}{\partial x_{1}} F(X)-\left(\frac{\partial}{\partial x_{1}} A(X)\right) Q
$$

for $1=0, \ldots, N$, where

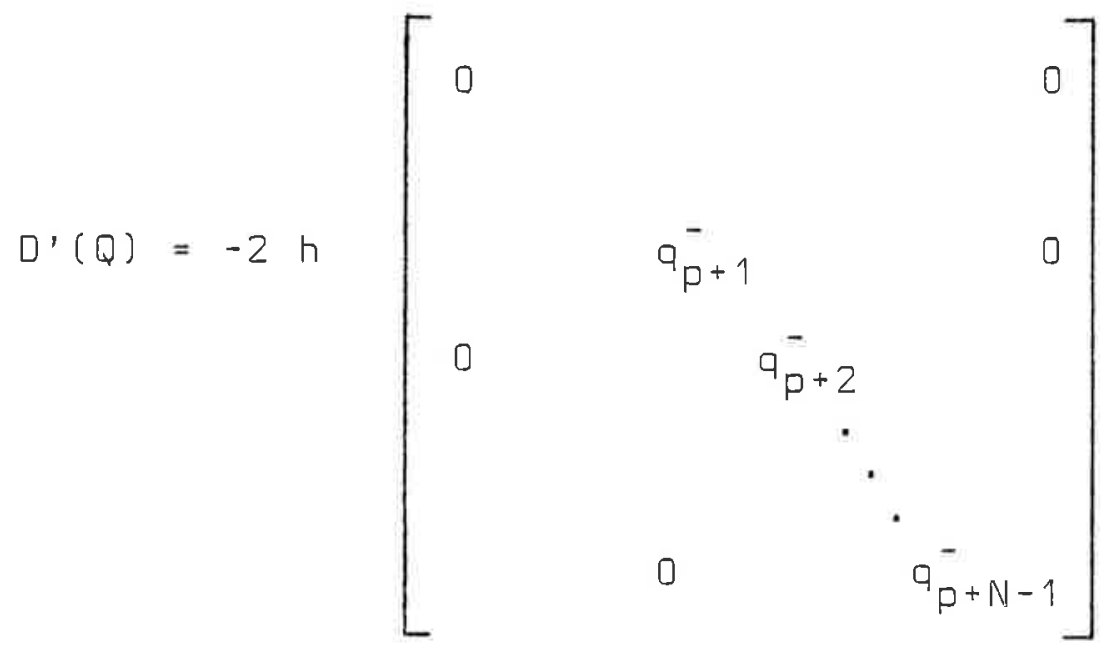

Thence, by $(4.10) \frac{\partial}{\partial x_{1}} Q(X)$ is obtained as a solution of linear system (4.13). As $A(X)$ is positive definite, the same is true for $\left(A(X)-\frac{1}{\varepsilon} D^{\prime}(Q)\right)$.

From the construction of $A(X)$ and $F(X)$ it is evident that the derivatives of $A(X)$ and $F(X)$ on the right hand side of $\{4.13$ \} must be computed from corresponding derivatives of the local stiffness matrix and of the local force vector. For details see [20].

4.4 Algorithms for solving Problem (P) $h$

The steps for solving the discretized shape design problem (P) are: solve the discrete state equation (3.2) in order to obtain an objective function $\psi\left(X, Q(X)\right.$ ), compute $\nabla_{X} \psi(X, Q(X))$ (if exists) and finally use an appropriate minimizing algorithm for finding a decreasing sequence: $\psi\left(X^{i}, Q\left(X^{i}\right)\right) \geq \psi\left(X^{i+1}, Q\left(X^{i+1}\right)\right)$. These steps are considered now in more detail. 
1. The state problem. Because of the nonlinear part $D(Q)$ the state equation (4.3) must be solved iteratively. Both gradient methods and relaxations methods can be applied. Due to the high dimension of the problem we have used the relaxation method.

The nonlinear over relaxation algorithm for solving (4.3) with the penalty term $\varepsilon$ reads:

Algorithm (NSOR) Let $Q^{0} \in \mathbb{R}^{K(h)}$ be arbitrarily chosen. Then $Q^{n}$ being known, compute $Q^{n+1}$, component by component as follows. Let

$(4.14)$

$$
q_{i}^{n+1 / 2}=\frac{1}{a_{i i}} G_{i}^{n+1} \text { for } i \notin I\left(\Gamma\left(v_{h}\right)\right) \text { or if } G_{i}^{n+1} \geq 0
$$

and

$$
q_{i}^{n+1 / 2}=\frac{a_{i i}-\left(a_{i i}^{2}-4 h \varepsilon^{-1} G_{i}^{n+1}\right)^{1 / 2}}{2 h / \varepsilon}
$$

for $i \in I\left(\Gamma\left(v_{h}\right)\right)$ and $G_{i}^{n+1}<0$, where

$$
G_{i}^{n+1}=F_{i}-\sum_{j=1}^{i-1} a_{i j} q_{j}^{n+1} \sum_{j=i+1}^{K(h)} a_{i j} q_{j}^{n}
$$

and where $I\left(\Gamma\left(v_{h}\right)\right)$ refers to indices $i=p+1, \ldots, p+N-1$ corresponding to nodes of $\Gamma\left(v_{h}\right)$. Set

$$
q_{i}^{n+1}=q_{i}^{n}+\omega\left(q_{i}^{n+1 / 2}-q_{i}^{n}\right) .
$$

The critical point of Algorithm (NSOR) is the optimal choice of relaxation parameter $\omega$. In practice $Q^{D}$ will be chosen to be equal to the solution of the state problem of the previous iteration in the minimization procedure (step 3 ). 
2. The gradient for cost functional. Form $\frac{\partial}{\partial x_{1}} F(X)$ and $\frac{\partial}{\partial x_{1}} A$ for $I=0, \ldots, N$ by using local force vectors and local stiffness matrixes. Solve equation (4.13) by cholesky method in order to obtain $\frac{\partial}{\partial x_{I}} Q(X)$ for $1=0, \ldots . N$. Compute $\frac{\partial}{\partial x_{I}} \operatorname{meas}(T)$, $T \in T_{h}\left(v_{h}\right)$ in order to calculate the term $\frac{\partial}{\partial x_{1}} b_{i j}(x), 1=0, \ldots, N$ (the majority of these terms will be identically zero). Pinally $\frac{\partial}{\partial x_{1}} \psi(X, Q(X))$ is then obtained by equation (4.10).

3. Minimizing of cost functional. In a concrete choice of the optimization algorithms, specific features of the problem have to be taken into account:

i) The evaluation of cost functional and its gradient are time consuming (steps 1 and 2 ). The Hessian matrix cannot be given explicitly..

ii) Constraints are linear containing box constraints, inequality constraints (Lipschitz-condition) and an equality constraint (meas $\left.\left(\Omega_{h}\left(v_{h}\right)\right)=c_{2}\right)$. The Lipschitz condition reads

$$
-\frac{C_{1}}{N} \leq x_{1}-x_{1-1} \leq \frac{C_{1}}{N}, \quad I=1, \ldots, N
$$

and the volume constraint

$$
\sum_{I=1}^{N}\left(x_{1}+x_{1-1}\right)=2 C_{2} N .
$$

This implies that matrix $\left(C_{i j}\right)$ in $(4.9)$ is sparse.

iii) Function $v \rightarrow J(y(v))$ is not convex. Thence an initial guess plays an important role in minimizing procedure. Because of the complexity and the high dimensionality of the problem global optimization is not relevant. 
For algorithms solving linearly constrained optimization problems we refer to $[8,9]$, to [18] (large scale linearly constrained problems) and to [30].

\section{REMARKS ON ALTERNATIVE METHODS}

\subsection{Dual formulation for the state problem}

By applying Green's formula to (2.4) one can easily prove that $y=y(v) \in K(\Omega(v))$ satisfies in $\Omega(v)$ the poisson equation with mixed Dirichlet-Signorini boundary conditions:

(5.1) $\left\{\begin{array}{rlrl}-\Delta y=f & \text { in } \Omega(v) \\ y=0 & \text { on } \Gamma_{1} \\ y \geq 0, \frac{\partial}{\partial n} y \geq 0, y \cdot \frac{\partial}{\partial n} y=0 \quad \text { on } \Gamma(v)\end{array}\right.$

For regularity of such type: of a problem see [2].

Using a saddle-point formulation to a unilateral boundary value problem (5.1) on $K(\Omega(v))$ ( $v \in u_{\text {ad }}$ being fixed) we obtain a problem: Find $\left(y, \frac{\partial}{\partial n} y\right) \in V(\Omega(v)) \times \Lambda$ such that

(5.2) $L\left(y, \frac{\partial}{\partial n} y\right)=\min _{\varphi \in V(\Omega(v))} \sup _{\mu \in \Lambda} L(\varphi, \mu)=\max _{\mu \in \Lambda} \inf _{\varphi \in V(\Omega(v))} L(\varphi, \mu)$,

where $L: V(\Omega(v)) \times \Lambda \rightarrow \mathbb{R}$ is a lagrangian defined by means of

$$
L(y(v), \mu)=\frac{1}{2}\|\operatorname{grad} y(v)\|_{0, \Omega(v)}^{2}-(f, y(v)) 0, \Omega(v)-\langle\mu, y(v)\rangle
$$

and

$$
\Lambda=\left\{\mu \in H^{-1 / 2}((0,1)) \mid \mu \geq 0\right\} .
$$


The symbol $<$, > denotes the duality pairing between $H^{-1 / 2}((0,1))$ and $H^{1 / 2}((0,1))$, which is an extension of a scalar product in $L^{2}((0,1))$, i.e.

$$
\begin{aligned}
& \langle\mu, y(v)\rangle=\int_{0}^{1} \mu\left(x_{2}\right) y\left(v\left(x_{2}\right)\right), d x_{2}, \\
& \mu \in L^{2}((0,1)) .
\end{aligned}
$$

By utilizing the classical Uzawa method to (5.2), we have the following iterative procedure for solving the original unilateral boundary value problem (5.1) in $\Omega(v)$ :

$\frac{\text { Algorithm 5.1. }}{y^{(k)} \in V(\Omega(v))}$ such that $\lambda^{0} \in \Lambda$ be given. If $\lambda^{(k)} \in \Lambda$ is known, find

$$
L\left(y^{(k)}, \lambda^{(k)}\right) \leq L\left(\varphi, \lambda^{(k)}\right) \text { for all } \varphi \in V(\Omega(V)) \text {. }
$$

Replace $\lambda^{(k)}$ by a new value $\lambda^{(k+1)}$ as follows

$$
\lambda^{(k+1)}=P_{\Lambda}\left(\lambda^{(k)}-\rho y^{(k)}\right), \rho>0,
$$

where $P_{\Lambda}$ is a projection onto the canvex set $\Lambda$.

The convergence of the above type of algorithm is studied in [4]. Step (5.3) consists of a mixed elliptic boundary value problem

$(5.5)$

$$
\begin{cases}-\Delta y(v)^{(k)}=f & \text { in } \Omega(v) \\ y(v)^{(k)}=0 & \text { on } \Gamma_{1} \\ \frac{\partial}{\partial n} y\left(v\left(x_{2}\right)\right)^{(k)}= & \frac{\lambda^{(k)}\left(x_{2}\right)}{\left(1+\left(v^{\prime}\left(x_{2}\right)\right)^{2}\right)^{1 / 2}}, x_{2} \in(0,1) .\end{cases}
$$


The algorithm we propose here for solving the optimal shape design problem (2.1) with the state problem (5.1) proceeds as follows:

Algorithm 5.2. Start with arbitrarily given $\lambda^{(0)} \in \Lambda$. Let $\lambda^{(k)} \in \Lambda$ be already known. Then solve the optimal shape design problem. (2.1) with (5.5) as a state equation.

Let $v^{(k)} \in u_{\text {ad }}$ be its solution and $y^{(k)}\left(v^{(k)}\right)$ be the corresponding state, respectively. Finally, replace $\lambda^{(k)}$ by $\lambda^{(k+1)}$ by means of

$$
\lambda^{(k+1)}=P_{\Lambda}\left(\lambda^{(k)}-\rho y^{(k)}\left(v^{(k)}\right)\right), \rho>0 .
$$

Let us note in this place that the convergence of Algorithm 5.2 remains still an open problem.

\subsection{Adjoint state for the penalized problem}

In chapter 4.4 the gradient for the cost functional $J_{\varepsilon}$ is computed in algebraic form. Due to the simple geometry it is quite straightforward. We note however on the use of more general methods in sensitivity analysis of shape design problems (material (or speed) derivatives, see [6], [29]). An essential point is to find the adjoint state. In connection of the penalized problem (2.5) it reads: Find $p \in V(\Omega(v))$ such that

$$
\begin{aligned}
& \int(v) \operatorname{grad} p \cdot \operatorname{grad} \xi d x+\left.\left.\left.\frac{2}{\varepsilon} \int_{0}^{1} p\right|_{\Gamma(v)} y_{\varepsilon}^{-}\right|_{\Gamma(v)} \xi\right|_{\Gamma(v)} d x_{2} \\
& =2 \underset{\Omega(v)}{\int}\left(y_{\varepsilon}-z_{d}\right) \xi d x \text { for all } \xi \in V(\Omega(v)) \text {. }
\end{aligned}
$$

By utilizing the adjoint state equation (5.6), the material derivative for cost functional $J_{\varepsilon}$ can be computed by evident modifications of methods presented in [6, 29]. See also [26, 27$]$. 
6. NUMERICAL EX்AMPLES

Problem ( $P$ ) has been solved numerically by using three different methods for the state problem:

(i) variational inequality formulation

(ii) method of penalization

(iii) dual formulation.

We consider the minimization of the functional for total displacements:

$(6.1)$

$$
J_{1}(v)=\int_{\Omega(v)}(y(v))^{2} d x
$$

as well as for boundary displacements:

$$
J_{2}(v)=\int_{0}^{1}(y(v))^{2} d x_{2}
$$

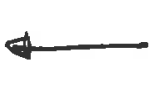

This cost functional

We have considered in the theoretical part only the case of the first cost functional, but the results are straightforward to extend to cover the second one as well.

The algorithms presented above were implemented to UNIVAC 1100/60 computer of Computer Centre of the University of Jyväskylä. In minimization module of design procedure the subroutine VEO1A of Harwell Subroutine Library was utilized. The authors are indebted to B.Sc. T. Tiihonen for his assistance in numerical experiments.

Example 6.1. In this example we have applied the above mentioned three different types of variational methods for minimizing the cost functional ( 6.1$)$ over $u_{\text {ad }}^{h}$ with $h=1 / 8$. 
Let $f\left(x_{1}, x_{2}\right)=4 \sin 2 \pi x_{2}$ and assume in the definition of $u_{\text {ad }}^{h}$ (see formula (4.6)) $\alpha=\frac{1}{2}, \beta=\frac{3}{2}, c_{1}=1, c_{2}=1$. The initial guess is $x^{0}=(1, \ldots, 1)$, i.e. $\Omega^{0}=(0,1) \times(0,1)$.

In variational inequality formulation difference approximation is used to provide $\nabla_{X} \psi_{1}(X, Q(X))\left(\psi_{1}(X, Q(X))\right.$ is the discretized form of $J_{1}(v)$, see chapter 4.2).

In penalty method and dual method $\nabla_{X} \psi_{1}(X, Q(X))$ was computed by formulae presented in chapter 4.3. Penalty parameter $\varepsilon$ was chosen to be $=10^{-6}$ and parameter $\rho$ in Uzawa algorithm $=5$. Single precision was utilized. The obtained results can be seen in Table 6.1.

\begin{tabular}{|c|c|c|c|}
\hline & $\begin{array}{l}\text { Variational } \\
\text { ineq. formul. }\end{array}$ & $\begin{array}{l}\text { Penalty } \\
\text { method }\end{array}$ & $\begin{array}{l}\text { Dual } \\
\text { method }\end{array}$ \\
\hline Initial value of $\psi_{1}$ & .0035647 & .0035645 & .00356710 \\
\hline Final value of $\psi_{1}$ & .0027075 & .0026696 & .0026683 \\
\hline $\begin{array}{l}x_{1} \text {-coordinates of } \\
\text { the unknown } \\
\text { boundary }\end{array}$ & $\begin{array}{r}1.0736472 \\
.9486468 \\
.8236468 \\
.9486468 \\
1.0736468 \\
1.1796846 \\
1.0546840 \\
.9296840 \\
1.0090733\end{array}$ & $\begin{array}{r}1.0631734 \\
.9381738 \\
.8131729 \\
.9381729 \\
1.0631734 \\
1.1866188 \\
1.0616407 \\
.9366406 \\
1.0616407\end{array}$ & $\begin{array}{r}1.0624992 \\
.9374992 \\
.8124990 \\
.9374999 \\
1.0625005 \\
1.1875005 \\
1.0825006 \\
.9375006 \\
1.0625005\end{array}$ \\
\hline $\begin{array}{l}\text { Number of gradient } \\
\text { evaluations (ite- } \\
\text { rations) }\end{array}$ & 36 & 31 & 28 \\
\hline $\begin{array}{l}\text { CPU-time is } \\
\text { seconds }\end{array}$ & 175 & 58 & 70 \\
\hline
\end{tabular}

Table 6.1. Comparision of different methods. 
It can be seen that the penalty method gives the best result. The higher CPU-time in variational inequality formulation is due to the fact that difference method is used for approximating $\nabla_{X} \psi_{1}(X, Q(X))$. Every iteration in this connection means ten function evaluations. The function evaluation in dual method is slightly more time consuming than in penalty method (UzawaNSORJ. In both methods the gradient of the cost functional is evaluated by methods presented in chapter 4.4 .

The solution of the state equation in penalty method with $\Omega^{31}$ (last iteration), the triangulation for $\Omega^{31}$ and the final position of design nodes $A_{i}=\left(x_{i}\right.$, ih) can be seen in Figure 6.2.

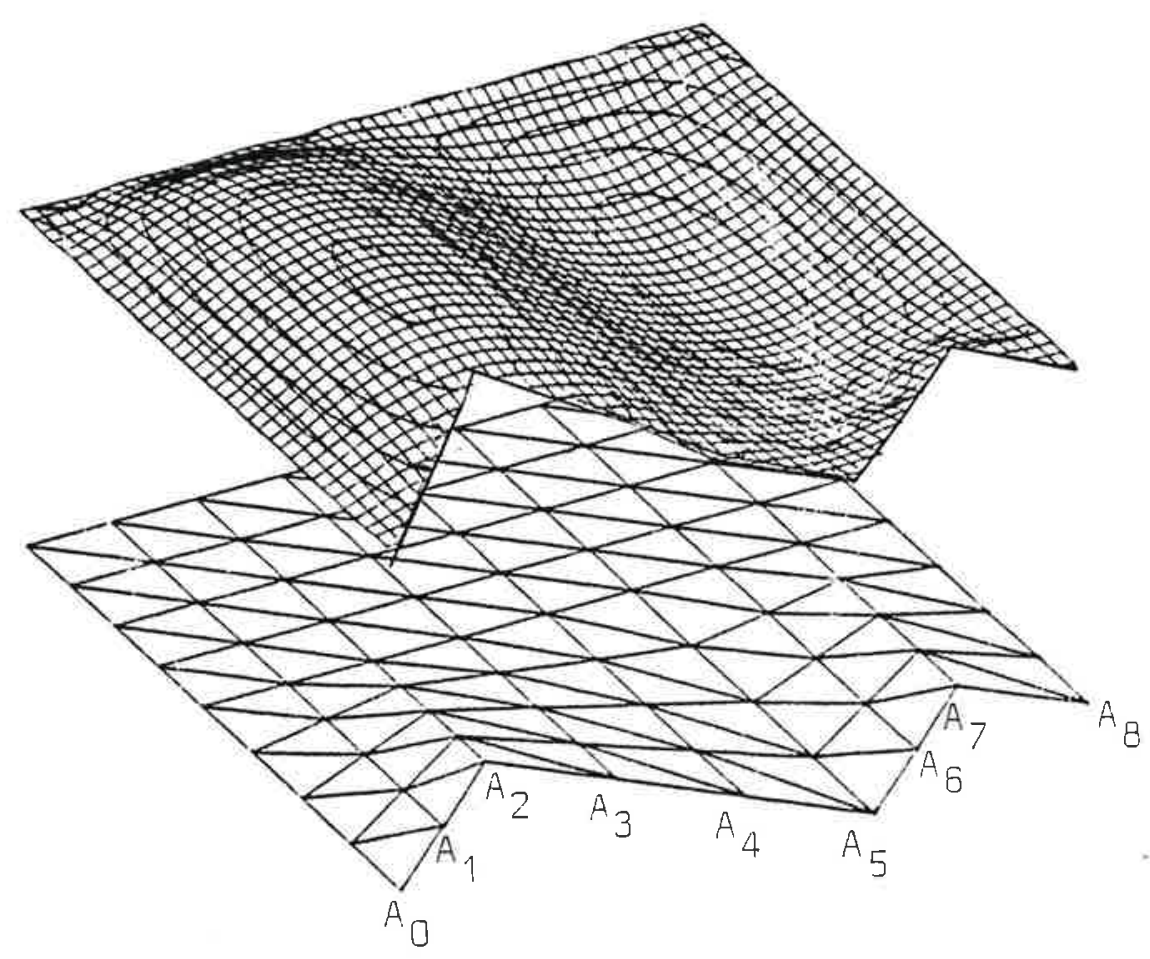

Figure 6.2. Spline-smoothed FE-solution of the state equation (3.2) with $\Omega^{31}$, triangulation of $\Omega^{31}$ and final position of design nodes $A_{i}(i=0, \ldots, 8)$.

For finer triangulation ( $h=1 / 16, h=1 / 32$ etc. $)$ the number of design variables is 17,33 etc. In optimization procedure the number of iterations for finding as good design as above remains almost the same. The function evaluation is however highly more time consuming: for example about 8 times more 
expensive for $h=1 / 16$ than for $h=1 / 8$. In this connection no essentially new information can be obtained by finer triangulation.

One possibility is to begin the design procedure with a rough triangulation and to take the obtained optimal design nodes plus nodes between these nodes as a new initial guess.

Example 6.2. In this example the criteria function is (6.2). Let

$$
f\left(x_{1}, x_{2}\right)=8 \sin 2 \pi x_{1} \sin 2 \pi x_{2} .
$$

We assume that $h=1 / 8, \alpha=3 / 4, \beta=3 / 2, C_{1}=1, C_{2}=1$.

As already mentioned, the criteria function is not convex, so that the initial guess has great influence for the design obtained when standard minimization routines are utilized (local minimum). To illustrate this situation let us consider three initial guesses for $x_{1}$-coordinates of design variables (see Table 6.3).

\begin{tabular}{|l|l|}
\hline $\begin{array}{l}x_{1} \text {-coordinates of design } \\
\text { variables }\end{array}$ & $\begin{array}{l}\text { value of criteria } \\
\text { function }(6.2)\end{array}$ \\
\hline$X_{1}^{0}=(1.0, .9, .8 ., 9,1.0,1.1,1.2,1.1,1.0)$ & $J_{2}=.31898 \mathrm{E}-3$ \\
\hline$x_{2}^{0}=(1.0,1.0,1.0,1.0,1.0,1.0,1.0,1.0,1.0)$ & $J_{2}=.38345 \mathrm{E}-2$ \\
\hline$X_{3}^{0}=(1.0,1.1,1.2,1.1,1.0, .9, .8, .9,1.0)$ & $J_{2}=.23762 \mathrm{E}-2$ \\
\hline
\end{tabular}

Table 6.3. Value of criteria function for different initial guesses.

In Table 6.4 we see the final values of the criteria functions for the above three initial guesses. Penalty method with $\varepsilon=10^{-6}$ is used for solving the state problem. 


\begin{tabular}{|c|c|c|c|}
\hline & Guess $x_{1}^{0}$ & Guess $x_{2}^{0}$ & Guess $x_{3}^{0}$ \\
\hline $\begin{array}{l}\text { Value of cost } \\
\text { function } J_{2} \text { for } \\
\text { initial guess }\end{array}$ & $.31898 \quad E-3$ & $.38345 E-2$ & $.23762 \quad E-2$ \\
\hline $\begin{array}{l}\text { Final value of } \\
\text { cost functional }\end{array}$ & $.24012 E-4$ & $.15546 \quad E-4$ & $.15238 E-2$ \\
\hline $\begin{array}{l}\text { Final } \times_{1}-\text { coor- } \\
\text { dinates of } \\
\text { design } \\
\text { variables }\end{array}$ & $\begin{array}{r}.7500000 \\
.7499999 \\
.8324477 \\
.9574477 \\
1.0811422 \\
1.2061422 \\
1.1228118 \\
1.1583375 \\
1.0333421\end{array}$ & $\begin{array}{r}.7500000 \\
.7500000 \\
.8234432 \\
.9484432 \\
1.0621496 \\
1.1871496 \\
1.2409772 \\
1.1158916 \\
.9918914\end{array}$ & $\begin{array}{l}1.0969671 \\
1.1935951 \\
1.1329431 \\
1.1250003 \\
1.0000002 \\
.8750000 \\
.7500000 \\
.8750000 \\
.9999559\end{array}$ \\
\hline $\begin{array}{l}\text { Values of } \\
\text { nodal dis- } \\
\text { placements } \\
\text { on final } \\
\text { design points } \\
\text { (principal } \\
\text { moving points) }\end{array}$ & $\begin{array}{r}.0000000 \\
.0000000 \\
-.0007669 \\
-.0009479 \\
.0004957 \\
.0126108 \\
-.0002110 \\
.0055680 \\
-.0003415 \\
.0000000\end{array}$ & $\begin{array}{r}.0000000 \\
.0000000 \\
-.0007498 \\
.0009452 \\
-.0005171 \\
.0113655 \\
-.0001061 \\
-.0007284 \\
.0004256 \\
.0000000\end{array}$ & $\begin{array}{r}.0000000 \\
.0000000 \\
.0135833 \\
.0088125 \\
.0026262 \\
-.0004898 \\
.0440756 \\
.0841414 \\
.0538423 \\
.0000000\end{array}$ \\
\hline $\begin{array}{l}\text { Number of } \\
\text { iterations }\end{array}$ & 60 & 60 & 19 \\
\hline $\begin{array}{l}\text { CPU-time } \\
\text { (seconds) }\end{array}$ & 77 & 76 & 23 \\
\hline
\end{tabular}

Table 6.4. Influence of initial guess for design obtained 
In Table 6.4 it can be seen that the first two initial guesses lead roughly speaking to the same minimum. For initial guess $x_{3}^{0}$ the algorithm can reduce the cost functional relatively less than for initial guess $x_{2}^{0}$ for example (47\% - $\left.99.9 \%\right)$. The final design is also different from that obtained for the other two initial guesses.

The solution of the state problem for $\Omega^{60}$ (with initial guess $\left.x_{2}^{0}\right)$, final triangulation and final position of design nodes $A_{i}=\left(x_{i}\right.$, ih) can be seen in Figure 6.6.

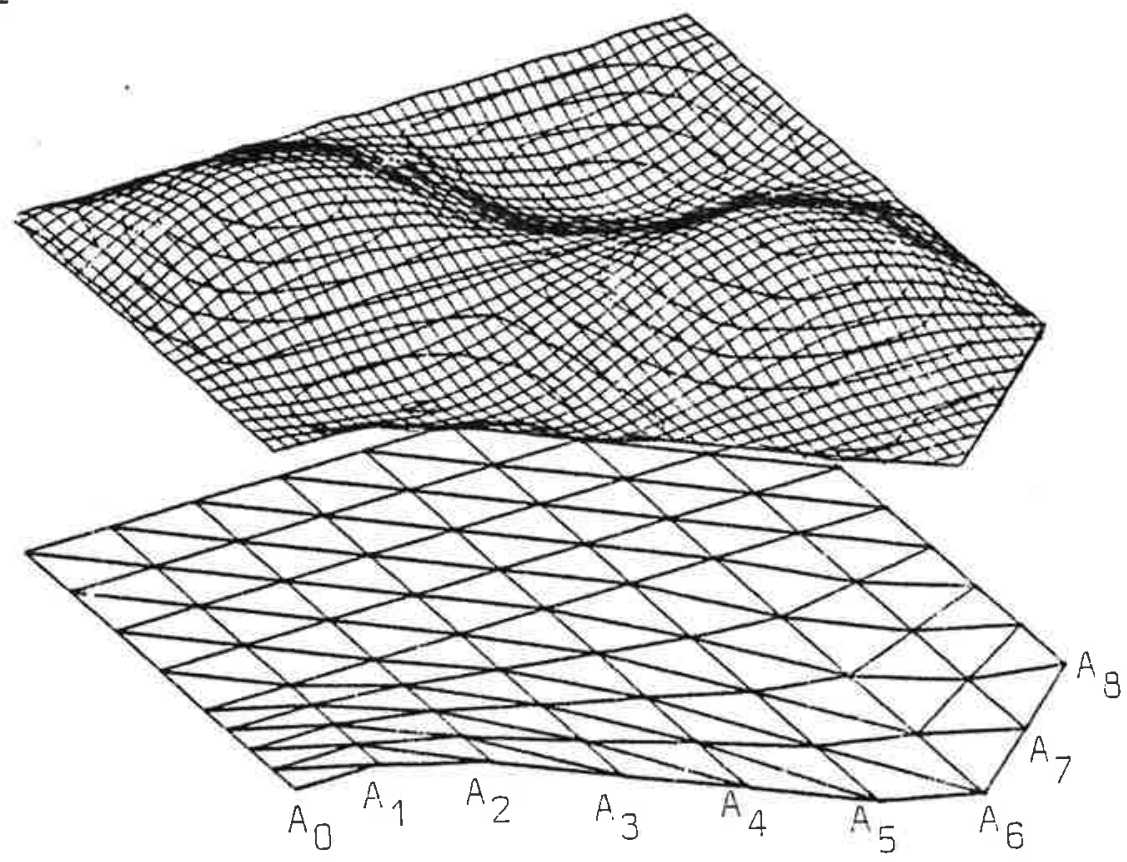

Figure 6.5. Spline-smoothed FE-solution of the state problem in Example 6.2. With $\Omega^{60}$ corresponding triangulation for $\Omega^{60}$ and final position of design nodes $A_{i}=i=0, \ldots, 8$.

It can be seen that the constraint $x_{1} \geq \alpha=3 / 4$ is active in the first two design nodes $A_{0}=(.75,0)$ and $A_{1}=(.75, .125)$. At node $A_{6} \approx(1.24, .75)$ the Lipschitz constraint is active. By choosing the constraints for $u_{\text {ad }}^{h}$ in an appropriate way we can find design points $A_{i}$ such that $u_{h}\left(A_{i}\right)=0, \frac{\partial}{\partial n} u_{h} \geq 0$. 
Example 6.1 as well as other methods for solving the state problem (variational inequality formulation and dual method) gave essentially the same "optimal design". The only difference is in CPU-time, which for penalty method and dual method is roughly speaking the same, but for variational inequality formulation about three times higher.

Finally, we consider the diminution of the criteria function $J_{2}$ in the design process. In Figure 6.6 we can see the decreasing of $J_{2}$ versus iterations; as above, the penalty method with $\varepsilon=1^{-6}$ for solving the state problem and the subroutine VEo1A of Harwell Subroutine Library for the minimization process were utilized. $\quad X_{2}^{0}$. was chosen as the initial guess (see Table 6.3).

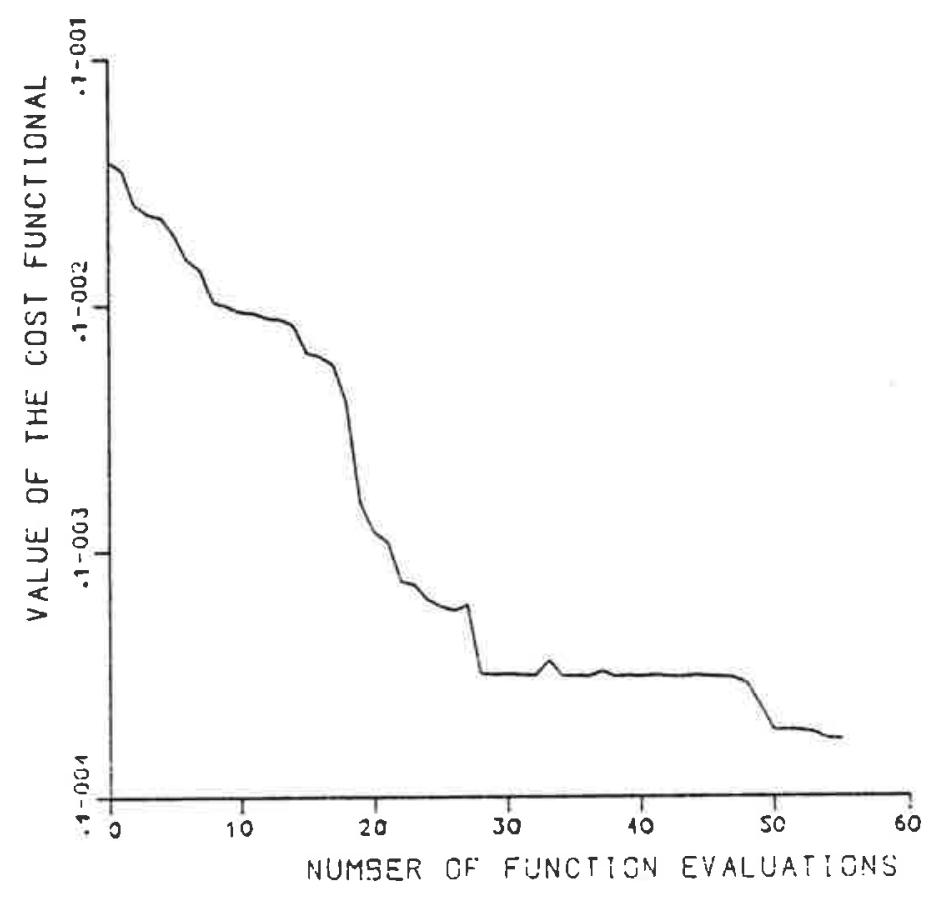

Figure 6.6. Decreasing of criteria function versus iteration

In Figure 6.6 the value of $J_{2}$ is given by a logarithmic scale. We can find that the criteria function has been reduced from $\Omega^{0}$ (corresponding to initial guess $x_{2}^{0}$ in Tabie 6.3) to $\Omega^{28}$ about a factor of 100 . From step $\Omega^{28}$ to $\Omega^{56}$ (28 iterations) the value of the criteria function has been reduced a relatively small amount. As the total cPU-time is here 76 seconds, about $50 \%$ of the CPU-time was used without obtaining any essential new information. 


\section{ACKNOWLEDGEMENT}

This research was supported by the Academy of Finland.

REFERENCES

[1] W.D. Arndt, Existenzsätze für Optimum Design Probleme bei Diffusionvorgängen, Dissertation, TH, Darmstadt, D 17,1978 .

[2] J. Bailet-Intissar, Non-linear mixed boundary value problems for elliptic partial differential equations, Proc. Roy. Soc. Edinburgh, 90 A (1981), 347-359.

[3] 0 . Begis and R. Glowinski, Application de la méthode des éléments finis à l'approximation d'un problème de domaine optimal, Appl. Math. Optim., 2 (1975), $130-169$.

[4] J. Cea, Optimization: Théorie et algorithmes, Dunod, Paris, 1971.

[5] J. Cea, Problems of shape optimal design, in [22] pp. $1005-1048$.

[6] J. Cea, Numerical methods of shape optimal design, in [22], pp. 1049-1088.

[7] G. Duvaut and J.L. Lions, Inequalities in mechanics and physics, Grundlenren der mathematischen Wissenschaften 219, Springer-Verlag, Berlin, 1976.

[8] R. Fletcher, Mathematical programming methods - a critical review, in Optimum Structural Design, Theory and applications, pp. 51-77, ed. R.H. Gallagher and o.C. Zienkiewicz, John Wiley \& Sons, London, 1973.

[9] R. Fletcher, Practical methods of optimization, vol. 2, constrained optimization, John Wiley \& Sons, Chichester, 1981. 
[10] J. Haslinger and P. Neittaanmäki, Penalty method in design optimization of systems governed by a unilateral boundary value problem, University of Jyväskylä, Dept. Math., Preprint 15, 1982.

[11] J. Haslinger and P. Neittaanmäki, On the method of penalization in design optimization of systems governed by some unilateral boundary value problems, University of Jyväskylä, Dept. Math., Preprint 18, 1983.

[12] J. Haslinger and $P$. Neittaanmäki, to appear.

[13] E.J. Haug, A review of distributed parameter structural optimization literature, in [22], pp. 3-68.

[14] E.J. Haug and J.S. Arora, Applied optimal design, mechanical and structural systems, Wiley - Interscience Publ., New York, 1979.

[15] I. Hlaváček and J. Nečas, Dptimization of the domain in elliptic unilateral boundary value problems by finite element method, R.A.I.R.O., Num. Anal., $16(1982), 351-373$.

[15] I. Hlaváček, I. Bock and J. Lovišek, Optimal control of variational inequalities with applications to structural analysis. Part I, optimal design of a beam with unilateral supports. Part II, Local optimization of the stress of a team. Part III, Optimal design of an elastic plate.

[17] F. Mignot, Contrôle dans les inéquations variationelles elliptiques, J. Funct. Anal., 22 (1976), 25-39.

[13] B.A. Murtagh, Large-scale linearly constrained optimization, Math. Programming, 14 (1978), 41-72.

[19] J. Nečas, Les Méthodes Directes en Thóorie des Equations Elliptiques, Masson, Paris, 1967.

[20] P. Neittaanmäki and T. Tiihonen, Optimal shape design of systems governed by a unilateral boundary value problem, Lappeenranta University of Technology, Dept. of Physics and Mathematics, Research Report 4/1982. 
[21] Dptimization Methods in Structural Design, Euromech Colloquium 164, [ed. by H. Eschenauer and N. Olhoff], Bibliographisches Institut Mannheim, 1983.

[22] Dptimization of Distributed Parameter Structures, [ed. by E.J. Haug and J. Cea], Nato Advanced Study Institutes Series, Series E, no. 49, Sijthoff \& Noordhoff, Alphen aan den Rijn, 1981.

[23] Dptimum Structural Design II, [ed. R.H. Gallagher], John Wiley \& Sons, New York, 1983.

[24] P.D. Panagiotopoulos, Optimal control in the unilateral thin plate theory, Arch. Mech. Stos., 29 (1977), 25-39.

[25] 0. Pironneau, Optimal shape design for elliptic systems, Pitman, 1983, to appear.

[26] J. Sokolowski, Sensitivity analysis of a class of variational inequalities, in [22], pp. 1600-1605.

[27] J. Sokolowski and J.P. Zolesio, Shape sensitivity analysis for variational inequalities, in Proceedings of 10th IFIP Conference, pp. 399-407, LN in Control and Information Sciences, 38, Springer-Verlag, Berlin, 1982 .

[28] W. Velte and P. Villaggio, Are the optimum problems in structural design well posed?, Arch. Mech. Anal. 78 (1982), pp. 200-211.

[29] J.P. Zolesio, The material derivative (or speed) method for shape optimization, in [22], pp. 1089-1151.

[30] G. Zoutendijk, Mathematical programming methods, NorthHolland Publishing Company, Amsterdam, 1976. 
UNIVERSITÄT JYVÄSKYLÄ

Mathematisches Institut

Preprints
UNIVERSITY OF JYVÄSKYLÄ

Department of Mathematics

Preprints

\section{L. KAHANPÄÄ}

Hypostetigkeit der Kompositionsabbildung in topologischen Operatorenidealen

(Preprint 1, 1979, 7 S.)

2. J. SARANEN

Über das Verhalten der Lösungen der Maxwellschen Randwertaufgabe in einigen nichtglatten Gebieten

(Preprint 2, 1980, 20 S.)

3. J. SARANEN

The finite element method for the electric field of Maxwell's boundary value problem in polygonal domains of plane

(Preprint 3, 1980, 20 pp.)

4. P. NEITTAANMÄKI and J. SARANEN

On the finite element approximation for Maxwell's problem in polygonal domains of plane

(Preprint 4, 1980, 13 pp.)

5. P. NEITTAANMÄKI and J. SARANEN

On finite element approximation of the gradient for solution of Poisson equation

(Preprint 5, 1980,6 pp.)

6. P. NEITTAANMÄKI and J. SARANEN

Finite element approximation of vector fields given by curl and divergence

(Preprint 6, 1980, 13 pp.)

7. P. NEITTAANMÄKI and J. SARANEN

Semi-discrete Galerkin approximation method applied to initial boundary value problems for Maxwell's equations in anisotropis, inhomogeneous media

(Preprint 7, 1980, 13 pp.)

8. P. NEITTAANMÄKI and J. SARANEN

Finite element approximation of electromagnetic fields in the three dimensional space

(Preprint 8, 1980, 24 pp.)

9. S. GRANLUND, P. LINDQVIST and O. MARTIO

Conformally invariant variational integrals

(Preprint 9, 1981, 61 pp.)

10. J. SARANEN

On an inequality of Friedrichs

(Preprint 10, 1981, 21 pp.)

11. J. SARANEN

On generalized harmonic fields in domains with anisotropic nonhomogeneous media

(Preprint 11, 1981, $15 \mathrm{pp}$. )

12. P. NEITTAANMÄKI and J. SARANEN

A mixed finite element method for the heat flow problem

(Preprint 12, 1981, 8 pp.) 
13. S. GRANLUND, P. LINDQVIST and O. MARTIO

F-harmonic measure in space

(Preprint 13, 1981, 24 pp.)

14. M. SAARIMÄKI

Counterexamples to the algebraic closed graph theorem (Preprint 14, 1981, 7 pp.)

15. J. HASLINGER and P. NEITTAANMÄKI

Penalty method in design optimization governed by a unilateral boundary value problem

(Preprint 15, 1982, 23 pp.)

16. S. GRANLUND, P. LINDQVIST and 0. MARTIO

Note on the PWB-method in the non-1 inear case

(Preprint 16, 1982, 21 pp.)

17. M. KR̈ IZ̈EK and P. NEITTAANMÄKI

On the validity of Friedrichs' inequalities

(Preprint 17, 1982, 16 pp.)

18. J. HASLINGER and P. NEITTAANMÄKI

On the method of penalization in design optimization of systems governed by some unilateral boundary value problems

(Preprint 18, 1983, 17 pp.)

19. T. KUUSALO

A direct proof of Smirnov's theorem on locally metrizable spaces (Preprint 19, 1983, 4 pp.)

20. K. ASTALA and H.-O. TYLLI

On the bounded compact approximation property and measures of noncompactness

(Preprint 20, 1983, 22 pp.)

21. S. GRANLUND, P. LINDQVIST and 0 . MARTIO

Phragmén-Lindelöf's and Lindelöf's theorems

(Preprint 21, 1983, 42 pp.) 